\title{
Rotating nonlinear magnetic islands in a tokamak plasma
}

\author{
A. I. Smolyakov and A. Hirose \\ Department of Physics and Engineering Physics, University of Saskatchewan, Saskatoon, \\ Saskatchewan S7N OWO, Canada \\ E. Lazzaro and G. B. Re \\ Istituto di Fisica del Plasma, CNR-EURATOM, Milan, Italy \\ J. D. Callen \\ Department of Nuclear Engineering and Engineering Physics, University of Wisconsin, \\ Madison, Wisconsin 53706-1687
}

(Received 23 September 1994; accepted 16 January 1995)

\begin{abstract}
The nonlinear dynamics of rotating low $m$ (poloidal mode number) tearing modes in a tokamak with external resonant magnetic perturbations is examined. Nonlinear evolution equations for the island width and the toroidal rotation frequency are derived within the two-fluid magnetohydrodynamic model, taking into account the plasma rotation and neoclassical parallel viscosity. The nonlinear stability of magnetic islands interacting with a static external magnetic perturbation is considered, and the critical magnetic field for the appearance of a locked mode is determined. It is shown that the coupling of the perpendicular and longitudinal plasma flow due to the neoclassical plasma viscosity enhances the amplitude of the critical magnetic field compared to the value obtained in a slab approximation. The perpendicular plasma viscosity causes a finite phase shift between the applied external field and the magnetic island, and further increases the value of the critical magnetic field required to induce a magnetic island. (C) 1995 American Institute of Physics.
\end{abstract}

\section{INTRODUCTION}

The problem of active stabilization of low $m$ (poloidal mode number) resistive magnetohydrodynamics (MHD) modes and avoidance of error field induced locked modes is of great importance for current tokamak research ${ }^{1-5}$ and may be crucial for the next generation of tokamak devices. ${ }^{6,7}$ These problems involve nonlinear interaction of a finite size magnetic island with external resonant magnetic perturbations. Such an interaction is inherently a dynamic process ${ }^{8}$; that is, a full, time-dependent nonlinear model of magnetic island behaviour in the presence of an external field is needed to satisfactorily model these phenomena. In this paper we derive Rutherford-type ${ }^{9,10}$ nonlinear dynamic equations for the evolution of the island width and the island rotation frequency, taking into account the effects of plasma inertia and transverse viscosity. ${ }^{11,12}$ We consider the general case of rotating magnetic islands in a tokamak plasma, and the plasma itself may also be rotating in the laboratory frame. Since the plasma rotation in the tokamak geometry is determined to a large extent by the neoclassical effects, we include the effect of neoclassical parallel viscosity in our nonlinear model for magnetic islands. Within the framework of this model we consider the damping of the island rotation, which is often observed for unstable magnetic islands in the Rutherford regime, and investigate the process of nonlinear excitation of magnetic islands by an external field. We determine the critical magnitude of the external field, which leads to a bifurcation of the equilibrium into the state with a finite island width. Such a process is believed to be responsible for the field error induced instabilities in tokamaks. ${ }^{13-18}$ We show that an enhancement of an effective plasma inertia due to neoclassical effects substantially increases the amplitude of the external magnetic field required to induce magnetic islands.
We consider a single helicity nonlinear tearing mode neglecting effects of mode coupling. We use two-fluid magnetohydrodynamic equations to describe the plasma dynamics in the nonlinear region near the rational surface. An ideal MHD equilibrium is assumed for the external (linear) region. The standard matching procedure ${ }^{9}$ is implemented to derive the Rutherford-type nonlinear equation for the evolution of the island width. The dynamics of a magnetic island is affected by the plasma inertia through the longitudinal current induced by the ion polarization drift. In a homogeneous plasma this yields a stabilizing effect, and a finite threshold occurs in the amplitude of the external field, leading to a magnetic island of finite width. However, in an inhomogeneous plasma, in which there is a gradient in the equilibrium ion pressure, the effect of ion finite Larmor radius (FLR) modifies the ion polarization drift, so that it may become destabilizing under certain conditions, which depend on the frequencies of the mode and plasma rotation. ${ }^{19}$

Nonlinear magnetic islands in tokamaks are experimentally observed to rotate in the laboratory frame. ${ }^{17,20}$ We assume that, in general, the island rotation is different from the rotation of the bulk plasma. The plasma velocity in the island region deviates strongly from the equilibrium profile in the absence of a magnetic island, so that the plasma inside the magnetic island is moving together with the island (at least in the direction perpendicular to the magnetic field lines), and the velocity profile relaxes to the equilibrium in the region away from the magnetic island. In our model we assume that the global profile of the plasma parameters such as density, temperature and electric field are not affected significantly by the island growth, so that away from the rational surface an equilibrium is not affected significantly by the magnetic island.

The amount of plasma involved in the island rotation. 
which moves together with the island, is self-consistently determined through the profile of the toroidal plasma velocity in the presense of a magnetic island. The profile of the "equilibrium" electrostatic potential is modified by the rotating magnetic perturbation. In the lowest order (ignoring effects of the order of $\rho_{i}^{2} / W^{2}$, where $\rho_{i}$ is the ion Larmor radius and $W$ is the island half-width), the electrostatic potential is such that the plasma flow is tangent to the perturbed magnetic surfaces. The resultant profile of the toroidal plasma velocity is determined from the radial momentum balance equation. We assume that the characteristic timc scale for the evolution of the magnetic island is longer than the ion-ion collision time, so that the poloidal plasma rotation is strongly damped by the neoclassical (parallel) ion viscosity. Then, the profile of the poloidal plasma velocity can be determined by the standard neoclassical expression with the ion temperature gradient measured relative to the perturbed flux surfaces.

The modification in the toroidal plasma velocity due to the magnetic island is proportional to the difference between the frequency of the mode rotation and toroidal plasma rotation calculated with respect to the equilibrium plasma parameters. The magnetic island has no effects on the profile of the toroidal plasma velocity when the island is rotating at a frequency equal to the local value of the equilibrium frequency of toroidal rotation. The effect of plasma inertia in the equation for the magnetic island growth is also proportional to the difference between these two frequencies. The strong damping of the poloidal plasma rotation leads to an increase of the effective plasma inertia in the perpendicular direction due to coupling of the plasma flow across the magnetic field with the longitudinal flow. ${ }^{21}$

The evolution of the island rotation is governed by the toroidal momentum balance equation. ${ }^{10}$ The external electromagnetic torque associated with the resonant magnetic perturbation and/or eddy current in a resistive wall is balanced against viscous damping due to the transverse plasma viscosity. ${ }^{22}$ The transverse plasma viscosity is assumed to be anomalous with an empirical viscosity coefficient. For unstable magnetic islands in the Rutherford regime, the toroidal momentum of the plasma involved in the island motion increases with its width, which leads to the linear slowdown of the rotation as the island grows in size (when the external force and viscosity are negligible).

We also study the situation where magnetic islands do not occur without an external field. In this case magnetic islands can be induced by a static perturbation from the error field. We investigate a mechanism for a nonlinear excitation of nonrotating magnetic islands (locked modes). Such magnetic islands still experience the stabilizing effect of the inertia due to the plasma flow in the laboratory rest frame, so that the island excitation has a threshold in the amplitude of an external field. ${ }^{2,6,14.23}$ The enhanced plasma inertia due to the neoclassical poloidal damping increases the threshold in the amplitude of the external field for a nonlinear instability of a magnetic island. We show that the toroidal "renormalization" of the effective plasma inertia is consistent with the intuitive momentum balance for the toroidal plasma motion with the magnetic island. The transverse plasma viscosity causes a finite phase shift between the applied perturbation and the magnetic island at the point of bifurcation, and further increases the critical magnetic field. The expressions for the critical magnetic field are derived for different values of the anomalous plasma viscosity.

For an ideally axisymmetric system, the neoclassical viscosity in the toroidal direction is identically zero. The helical magnetic perturbation superimposed onto the axisymmetric tokamak configuration destroys the toroidal symmetry and creates a viscous force in the toroidal direction due to neoclassical (longitudinal) viscosity. This causcs an additional damping of the island rotation. We consider this effect using the analogy between the structure of the magnetic fields in a stellarator and a tokamak with a helical magnetic perturbation due to the magnetic island.

To model the situation typical for feedback control of magnetic islands, we assume that the external helical magnetic field can be rotating in the laboratory frame. In general, the frequency of island rotation is not equal to the frequency of rotation of an external field; neither is it constant in time. To investigate the evolution of the frequency of island rotation, we allow for an arbitrary time dependence in the phase of the magnetic island and in the phase of an external magnetic perturbation (which could be either rotating in the case of feedback stabilization, or static in the case of an error field). It will be shown that the perturbation of the magnetic field registered in the vacuum region at the plasma boundary consists of three principal parts: one is associated with the value of the reconnected flux at the rational surface (and thus it has phase information about island rotation), another part is proportional to the magnitude of the external field (this part is correlated in phase with the external current), and the third part represents the signal corresponding to the first two parts, which is reflected from the conducting wall. For a wall of finite resistivity the phase of the reflected signal has a finite time delay with respect to the part of the magnetic flux induced by an external current and the reconnected flux at the rational surface. This finite time delay is important in the dynamics of the phase instability of the magnetic island. The effect of finite plasma current in the region between the rational surface and the plasma boundary on the the structure of the perturbed flux function is investigated.

The paper is organized as follows. In Section II we review the basic theory of nonlinear island growth in the Rutherford regime for an inhomogeneous plasma. In Section III we consider the effects of toroidicity and investigate the dynamics of mode rotation. Here we derive the basic evolution equations for the island growth and rotation frequency. In Section IV the structure of the magnetic perturbation in the outer (linear) region is analyzed. In Section $V$ the problems of slowing-down of the island rotation in the Rutherford regime and the nonlinear excitation of locked modes are addressed. The results are discussed and summarized in Section VI.

\section{BASIC MODEL OF NONLINEAR ISLAND GROWTH IN A SLAB MODEL}

First, we consider the basic nonlinear model of nonlinear island growth in the slab approximation. Such an approxima- 
tion is relevant for the narrow magnetic islands in a cylindrical tokamak, where $W \ll a$ ( $a$ is the minor plasma radius). This model is mainly based on the Rutherford theory of nonlinear islands modified by the effect of plasma inertia, which is important for the rotating islands. ${ }^{11}$ The importance of plasma inertia on the dynamic behaviour of nonlinear tearing modes has been pointed out in Ref. 24 .

We consider a helical magnetic perturbation $(m, n$ are the poloidal and toroidal wave numbers) moving across the equilibrium magnetic field. Such a perturbation is resonant at the rational surface $r=r_{s}$, which has the same helicity $q\left(r_{s}\right)=m / n$. In the vicinity of the resonant surface, the magnetic field can be described by the effective helical flux function,

$$
\psi(x, t)=-\frac{x^{2}}{2 L_{s}} B_{0}+\tilde{\psi}(t) \cos \xi .
$$

Here, $x=r-r_{s}$ is the distance from the corresponding rational surface, $L_{s}=q R / S$ is the shear length, $S=q^{\prime} r_{s} / q$, and $B_{0}$ is the equilibrium toroidal magnetic field. The helical coordinate $\xi$ is given by the expression

$$
\xi=m \hat{\theta}-\int^{t} \omega\left(t^{\prime}\right) d t^{\prime},
$$

where $\hat{\theta}=\theta-\zeta / q_{s}$ is the helical angle in the direction perpendicular to the equilibrium magnetic field. The function $\tilde{\psi}(t)$ is taken to be independent of $x$ (constant $\Psi$ approximation").

Magnetic flux in the form (1) corresponds to a magnetic island with half-width $W$,

$$
W^{2}=\frac{4 L_{s} \tilde{\psi}}{B_{0}} \text {. }
$$

The nonlinear equations for the evolution of the magnetic island follow from the matching conditions obtained by integration of the Ampère's law, $4 \pi J_{\|} / c=\nabla_{\perp}^{2} \psi$, across the nonlinear region. The corresponding matching equations have the form ${ }^{25}$

$$
\begin{aligned}
& \int_{-\pi}^{\pi} d(m \hat{\theta}) \int_{-\infty}^{\infty} d x J_{\|} \cos \xi=\frac{c}{4 \pi} \Delta_{c}^{\prime} \tilde{\psi}, \\
& \int_{-\pi}^{\pi} d(m \hat{\theta}) \int_{-\infty}^{\infty} d x J_{\|} \sin \xi=\frac{c}{4 \pi} \Delta_{s}^{\prime} \tilde{\psi},
\end{aligned}
$$

where the matching parameters $\Delta_{c}^{\prime}$ and $\Delta_{s}^{\prime}$ are to be determined from the outer (linear) region. The longitudinal current $J_{\|}$in Eqs. (4) and (5) is determined from the nonlinear region.

The time evolution of the magnetic island is controlled by the longitudinal current induced in a plasma with finite resistivity by the inductive electric field associated with the time dependence in $\bar{\psi}(x, t)$. In the Rutherford theory the plasma inertia is neglected and the total plasma current has only the parallel component (along the magnetic field), which requires that the longitudinal current is a surface function of the magnetic flux. In this case the nonlinear island growth is merely a resistive steady-state evolution (relaxationl to a magnetir island-tune enuilihrium It has heen pointed out in Ref. 24 that the effect of plasma inertia provides a stabilizing effect on growing magnetic islands at an early stage of the nonlinear instability. The effect of plasma inertia can be even more important for a rotating island in an inhomogeneous plasma, where it is modified by the ion FLR and becomes destabilizing under certain conditions, leading to the possibility of nonlinear self-sustainment of magnetic islands. ${ }^{11,19}$ The plasma inertia manifests itself through the ion polarization drift, which creates a perpendicular current, contributing to the quasineutrality condition. In this case, the current closure equation is reduced to the form ${ }^{19,26,27}$

$\mathbf{b} \cdot \nabla\left(\frac{J_{\|}}{B}\right)-\frac{c^{2}}{4 \pi v_{A}^{2}} \frac{d_{0}}{d t} \nabla_{\perp}^{2} \Phi-\frac{e c}{m_{i} \omega_{B i}^{2} B_{0}} \nabla(\mathbf{b} \cdot \nabla \Phi \times \nabla) \cdot \nabla p_{i}$

$$
+\frac{c^{2}}{4 \pi v_{A}^{2}} \mu \nabla_{\perp}^{4}\left(\Phi+\frac{p_{i}}{e n_{0}}\right)=0,
$$

where $\mathbf{b}$ is the unit vector along the total magnetic field, $\Phi$ is the electrostatic potential, $p_{i}$ is the ion pressure and $\mu=3 T_{i} \nu_{i} /\left(10 m_{i} \omega_{B i}^{2}\right)$ is the magnetic viscosity coefficient. ${ }^{28}$ The third term in Eq. (6) is the correction to the ion polarization current due to the ion gyroviscosity by Braginskii, ${ }^{28}$ and the last one is due the collisional magnetic viscosity. We have assumed a homogeneous magnetic field in the equilibrium.

The operator $d_{0} / d t$ is given by the expression

$\frac{d_{0}}{d t}(\cdots) \equiv\left(\frac{\partial}{\partial t}+\mathbf{V}_{E} \cdot \nabla\right)(\cdots)=-\omega \psi_{x}\left\{x-\frac{k_{\theta} c}{\omega B_{0}} \Phi,(\cdots)\right\}$,

where $\{A, B\}$ is the Poisson bracket,

$$
\{A, B\}=\frac{\partial A}{\partial \psi} \frac{\partial B}{\partial \xi}-\frac{\partial A}{\partial \xi} \frac{\partial B}{\partial \psi} .
$$

The perpendicular current in Eq. (6) associated with the ion polarization drift and plasma viscosity creates the longitudinal current, which affects the island growth through Ampère's law in Eqs. (4) and (5). To calculate the longitudinal current we need to determine the perturbed ion pressure and electrostatic potential in Eq. (6). They can be calculated from the equations of two-fluid magnetohydrodynamics, taking into account the dynamics of the electron component (Appendix). In fact, in the small Larmor radius approximation, $\rho_{i} \ll W$, the ion pressure in Eq. (6) can be determined from the equation

$$
\frac{d_{0}}{d t} p_{i}=0 .
$$

This equation means that, in the steady-state approximation, $[(1 / \omega) \partial \omega / \partial t \approx(1 / W) \partial W / \partial t \ll \omega]$, the total ion pressure is a surface function $p=p\left(\Phi^{*}\right)$ of the electrostatic potential $\Phi^{*}$ in the reference frame moving with the island, $\Phi^{*}=\Phi-\left(\omega B_{0} / k_{\theta} c\right) x$. Within our model (Appendix), we choose this function so that the linear boundary conditions are satisfied away from the island:

$$
p_{i}=p(0)+p_{i 0}^{\prime}\left(x-\frac{k_{\theta} c}{\left(\omega-\omega_{F}\right) B_{0}} \tilde{\Phi}\right),
$$


where $\omega_{E}=k_{\theta} c \Phi_{0}^{\prime} / B_{0}$ is the drift frequency due to the equilibrium radial electric field $E_{r}=-\Phi_{0}^{\prime}$, and $\tilde{\Phi}$ is the perturbed electrostatic potential.

The electrostatic potential around the magnetic island is perturbed by the inductive electric field created by the magnetic perturbation moving across the equilibrium magnetic field. In steady state, the electrostatic component of the parallel electric field is almost balanced by the inductive electric field, so that the electrostatic potential can be found from the condition

$$
E_{\|}=-\nabla_{\|} \Phi+\frac{\omega}{c} \tilde{\psi} \sin \xi \simeq 0 .
$$

Here $\nabla_{\|}$is the nonlinear gradient operator along the total magnetic field given by the expression

$$
\nabla_{\|}=\frac{\mathbf{B} \cdot \nabla}{B_{0}}=\frac{k_{\theta}}{B_{0}} \frac{\partial \psi}{\partial x}\left(\frac{\partial}{\partial \xi}\right)_{\psi},
$$

where $k_{\theta}=m / r$ and the derivative $(\partial / \partial \xi)_{\psi}$ is taken at a constant $\psi$.

Using the identity

$$
\frac{\tilde{\psi} \sin \xi}{\psi_{x}}=\left(\frac{\partial x}{\partial \xi}\right)_{\psi},
$$

we obtain for $\Phi$,

$$
\Phi=\frac{\omega B_{0}}{k_{\theta} c} x+f(\psi),
$$

where $f(\psi)$ is an arbitrary function of $\psi$, which should be determined from the boundary conditions. ${ }^{19}$ The first term on the right of Eq. (13) corresponds to the electric field created by the moving magnetic field, so that after transformation to the reference frame in which the island is stationary, the electrostatic potential becomes a surface function of $\psi$. Thus, the plasma flow due to the $\mathbf{E} \times \mathbf{B}$ drift resulting from such an electrostatic field is tangent to the magnetic field and does not pierce the magnetic surfaces. In fact, Eq. (13) describes the electrostatic potential in one-fluid MHD approximation, in which the difference in the motion of the electron and ion components is ignored. In two-fluid magnetohydrodynamics, the electrostatic potential has to be determined selfconsistently from the quasineutrality condition, taking into account the finite ion current across the magnetic field. For perturbations with a perpendicular length scale larger than the ion Larmor radius, the corresponding quasineutrality equation can be considered perturbatively, so that Eq. (13) gives the electrostatic potential with an accuracy of the order of $\rho_{i}^{2} / W^{2}$ (Appendix). As is shown in the Appendix, the profile of the perturbed electrostatic potential around the magnetic island is given by

$$
\Phi=\frac{\left(\omega-\omega_{E}\right) B_{0}}{k_{\theta} c}[x-\lambda(\psi)] .
$$

Then. expression (9) for the total ion pressure takes the form

$$
p_{i}=p(0)+p_{i 0} \prime \lambda(\psi) \text {. }
$$

The plasma pressure is assumed to be flattened within the island and approaches the equilibrium profile in the re- gion away from the island, so that function $\lambda(\psi)$ is chosen to be $\lambda(\psi)=0$ inside the separatrix of the magnetic island and $\lambda(\psi) \rightarrow x$ in the region $x \gg W$, so that we have $p_{i}^{\prime} \rightarrow p_{i 0}^{\prime} x$ for large $x$. The precise form of the $\lambda(\psi)$ function in the outer region close to the separatrix is not very important [different forms of $\lambda(\psi)$ lead to slightly different numerical coefficients]; however, it is important to keep $\lambda(\psi)$ a single-scale function of $\psi$ with the characteristic length scale $W$.

Using Eqs. (14) and (15) in the current closure equation (6), after integration, we find for the parallel current, ${ }^{19}$

$$
\begin{aligned}
J_{\|}^{(0)}= & \frac{c}{8 \pi v_{A}^{2}} \frac{B_{s}^{4} W^{2}}{k_{\theta}^{2} L_{s}^{2}} \omega^{\prime}\left(\omega^{\prime}-\omega_{* p i}\right) \frac{\partial \lambda}{\partial \psi} \frac{\partial^{2} \lambda}{\partial \psi^{2}}(\cos \xi-\langle\cos \xi\rangle) \\
& +\frac{\sigma_{\|}}{c} \frac{\partial \tilde{\psi}}{\partial t}\langle\cos \xi\rangle,
\end{aligned}
$$

where $\omega^{\prime}=\omega-\omega_{E}$ and $\omega_{* p i}$ is the ion diamagnetic frequency, $\omega_{* p i}=k_{\theta} c T_{i} p_{0 i}^{\prime} /\left(e B p_{0 i}\right)$. The operator $\langle\cdots\rangle$ means averaging over the perturbed magnetic surface,

$$
\langle\cdots\rangle=\oint \frac{(\cdots) \psi_{x}^{-1} d \xi}{\psi_{x}^{-1} d \xi} .
$$

The first term in expression (16) corresponds to the current induced by the ion polarization drift and the second term is the Rutherford current induced by the inductive electric field. The contribution of the perpendicular collisional viscosity to the longitudinal current is odd in the leading order in $\xi$ and, therefore, omitted in Eq. (16), because it does not appear in the matching equation (4). As was noted in Ref. 19, in the leading order dissipative effects contribute to the second matching equation (5), and do not appear in the $\cos \xi$ hatmonic of the longitudinal current. Formally, it is related to the even parity of the function $\lambda(\psi)$ assumed in our analysis. Higher order corrections to $\lambda(\psi)$ (for instance those related to the asymmetric flow inside the magnetic island ${ }^{12}$ ) destroys this parity, so that the higher order effects of the perpendicular viscosity could appear in Eq. (16). They lead to second order effects in the equation for island growth, ${ }^{12}$ which are quadratic in $\mu$ (viscosity coefficient). These bigher order terms are neglected in our work.

Using (16) in the matching condition (4), we obtain the equation for the nonlinear growth of the magnetic island in a slab model,

$$
G_{1} \frac{\partial W}{\partial t}=D_{R}\left(\frac{\Delta_{c}^{\prime}}{4}-\frac{G_{3}}{W^{3}} L_{s}^{2} \frac{\omega^{\prime}\left(\omega^{\prime}-\omega_{* p i}\right)}{k_{\theta}^{2} v_{A}^{2}}\right),
$$

where $D_{R}=c^{2} / 4 \pi \sigma_{\|}, G_{1} \simeq 0.41^{9}$ and $G_{3} \simeq 1.06 .^{11}$ The first term on the right of this equation represents the standard Rutherford growth, and the second one corresponds to the effects of plasma inertia and ion FLR. ${ }^{11,12,19}$

\section{MAGNETIC ISLAND DYNAMICS IN TOROIDAL GEOMETRY}

\section{A. Effects of the parallel neoclassical viscosity on the island growth}

In toroidal geometry the plasma flow perpendicular to the equilibrium magnetic field couples to the longitudinal flow through the neoclassical parallel viscosity. A strong 
damping of the poloidal plasma flow causes an enhancement in the perpendicular dielectric constant that can be attributed to the effective renormalization of the ion mass in the ion polarization current. ${ }^{21}$ To describe this effect, the current closure equation (6), that controls the parallel current, should include a neoclassical parallel viscosity $\mathbf{I}_{\|}$term. As will be shown below, the effect of the neoclassical parallel viscosity dominates over the other inertial effects, so that the standard ion polarization current can be omitted. In the approximation of narrow magnetic islands, $\partial / \partial r \gg m / r_{s}$, the contribution of the parallel viscosity to the quasineutrality equation (6) can be transformed to the following form: ${ }^{21}$

$$
\frac{c}{B_{0}} \nabla \cdot\left(\mathbf{b} \times \nabla \cdot \mathbf{\Pi}_{\|}\right) \simeq-\frac{c}{B_{0} B_{\theta}} \frac{\partial}{\partial r}\left(\mathbf{B} \cdot \nabla \cdot \mathbf{\Pi}_{\|}\right) .
$$

This relation shows how the perpendicular plasma dynamics in a toroidal geometry couples to the parallel flow. The dynamics of the parallel ion motion is described by

$$
\begin{aligned}
m_{i} n_{0}\left(\frac{\partial}{\partial t}+\mathbf{V}_{E} \cdot \nabla\right) V_{\|} B= & -(\mathbf{B} \cdot \nabla) p-\mathbf{B} \cdot \nabla \cdot \mathbf{\Pi}_{\|} \\
& +m_{i} n_{0} \mu_{a} \frac{\partial^{2}}{\partial r^{2}} V_{\|} B
\end{aligned}
$$

where a phenomenological viscosity $\mu_{a}$ is introduced to describe an anomalous loss of the parallel momentum.

Within an accuracy of the order of $\epsilon=r / R$, the velocity of the plasma tlow along the magnetic field is approximately equal to the toroidal velocity, $V_{5} \simeq V_{\|}$, which can be found from the radial projection of the momentum balance equation for the ion component,

$$
V_{\zeta}=V_{\theta} \frac{B_{\zeta}}{B_{\theta}}-\frac{c}{B_{\theta}} \frac{\partial}{\partial r}\left(\Phi+\frac{p_{i}}{e n_{0}}\right)
$$

where, as usual, the radial inertial and viscous terms are neglected. Using Eqs. (18)-(20), we rewrite the current closure equation (6) in the form

$$
\begin{gathered}
\mathbf{B} \cdot \nabla\left(\frac{J_{\|}}{B}\right)-\frac{c^{2}}{4 \pi v_{a \theta}^{2}} \frac{\partial}{\partial r}\left(\frac{\partial}{\partial t}+\mathbf{V}_{E} \cdot \nabla+\mu_{a} \frac{\partial^{2}}{\partial r^{2}}\right) \\
\times\left[\frac{\partial}{\partial r}\left(\Phi+\frac{p_{i}}{q n_{0}}\right)-\frac{V_{\theta}}{c} B_{\zeta}\right]=0,
\end{gathered}
$$

where $v_{A \theta}=B_{\theta} / \sqrt{4 \pi n_{0} m_{i}}$ is the poloidal Alfvén velocity. We have neglected the Ware pinch term in Eq. (21), taking into account the fact that in our model the plasma pressure is constant on the perturbed magnetic surface, $p=p(\psi)$.

As mentioned in the Introduction, we assume that the ion-ion collision time is short compared to the characteristic time scale of the island growth, so that the poloidal plasma flow can be taken in the standard neoclassical form (in the relevant collisionality regime) with a temperature profile modified by the presence of a magnetic island,

$$
V_{\theta}=-k \frac{c}{a_{i} B_{r}} \frac{\partial T_{i}}{\partial r}
$$

where $k=-1.17$ in the banana regime. ${ }^{29}$ In the lowest order in the small parameter $\rho_{i}^{2} / W^{2}$, the profile of the ion temperature around the magnetic island can be taken in the form (see the Appendix)

$$
T_{i}=T_{i 0}^{\prime} \lambda(\psi)
$$

The electrostatic potential and ion pressure in Eq. (21) are defined by Eqs. (14) and (15). After integration, Eq. (21) is reduced to a form similar to equation (16),

$$
\begin{aligned}
J_{\|}^{(0)}= & \frac{c}{8 \pi v_{A \theta}^{2}} \frac{B_{0}^{4} W^{2}}{k_{\theta}^{2} L_{s}^{2}}\left(\omega-\omega_{E}\right)\left(\omega-\omega_{T}\right) \frac{\partial \lambda}{\partial \psi} \frac{\partial^{2} \lambda}{\partial \psi^{2}} \\
& \times(\cos \xi-\langle\cos \xi\rangle)+I(\psi),
\end{aligned}
$$

where $\omega_{T}=\omega_{E}+\omega_{* p i}+k \omega_{* T}$ is the equilibrium "neoclassical" toroidal rotation frequency, with $\omega_{* T}=k_{\theta} c T_{i}^{\prime} / e B_{0}$, and the surface function $I(\psi)$ is to be determined from the selfconsistency condition for the Ohm's law. In the toroidal case the parallel component of the electron momentum balance is modified by the effect of the neoclassical electron viscosity, leading to an additional driving mechanism for nonlinear mode growth due to a perturbed bootstrap current. ${ }^{30}$ Employing matching equation (4) with Eq. (24), we obtain

$$
\begin{aligned}
G_{1} \frac{\partial W}{\partial t}= & D_{R}\left(\frac{\Delta_{c}^{\prime}}{4}+\sqrt{\epsilon} G_{2} \frac{L_{s}}{L_{p}} \frac{\beta_{\theta}}{W}\right. \\
& \left.-\frac{G_{3}}{W^{3}} L_{s}^{2} \frac{\left(\omega-\omega_{E}\right)\left(\omega-\omega_{T}\right)}{k_{\theta}^{2} v_{A \theta}^{2}}\right),
\end{aligned}
$$

where $L_{p}=-(\partial \ln p / \partial r)^{-1}$ is the characteristic length scale of the pressure gradient, $\beta_{\theta}=8 \pi p / B_{\theta}^{2}, \epsilon=r_{s} / R$ and $G_{2}$ is the numerical coefficient of order unity. The second term on the right of the Eq. (25) is due to the perturbed bootstrap current. Equation (25) essentially has the same structure as Eq. (17) except for the additional term related to the bootstrap current. Coupling of the perpendicular and parallel flows in toroidal geometry leads to the renormalization of the Alfvén velocity in the last term in. Eq. (25), $v_{A}^{2} \rightarrow v_{A \theta}^{2}=v_{A}^{2} r_{s}^{2} / q^{2} R^{2}$.

\section{B. Momentum balance and island rotation}

The evolution of the island rotation is determined by the momentum balance in the nonlinear region. ${ }^{10}$ The toroidal component of the momentum balance equation is

$$
\begin{aligned}
m_{i} n_{0} \frac{d}{d t} \mathbf{B}_{\zeta} \cdot \mathbf{V}= & \frac{1}{c} \mathbf{B}_{\zeta} \cdot \mathbf{J} \times \mathbf{B}-\left\langle\mathbf{B}_{\zeta} \cdot \nabla \cdot \mathbf{\Pi}_{\|}\right\rangle \\
& +\mu_{a} m_{i} n_{0} B_{0} \nabla_{\perp}^{2} V_{\zeta} .
\end{aligned}
$$

The dynamics of plasma rotation is determined from the balance of the electromagnetic force, the neoclassical viscosity force and the anomalous loss of toroidal momentum, which is modeled here by the perpendicular viscosity with a phenomenological viscosity coefficient $\mu_{a}$. A similar term could also appear in the poloidal momentum balance. However, we assume that the neoclassical viscous force is the 
dominant term in the poloidal momentum balance, so that the poloidal velocity is not changed much by the presence of an island, and it is described by expression (22). In a tokamak, the toroidal component of the viscous force vanishes identically due to the toroidal symmetry, $\left\langle\mathbf{B}_{\zeta} \cdot \nabla \mathbf{I}_{\|}\right\rangle=0$. With the helical magnetic perturbation imposed on the tokamak equilibrium, the magnetic field is no longer axisymmetric and an additional neoclassical viscous force appears in the toroidal momentum balance. We consider this effect in the following section.

The evolution of the toroidal velocity on the right of Eq. (26) is associated mainly with the dynamics of the electrostatic potential. Within our model, the evolution of the electrostatic potential (13) is related to the time dependence in the frequency of the mode rotation $\omega$. Thus, Eq. (26) essentially describes the evolution of the mode frequency. Note that the first term in Eq. (14) for the electrostatic potential also contributes to the poloidal flow velocity, so that, in general, the evolution of $\omega$ affects the poloidal velocity as well. However, due to a strong (on the ion-ion time scale) damping of the poloidal rotation, the change in the poloidal velocity associated with the evolution of $\Phi$ is compensated by the change in the parallel flow velocity $V_{\|}$, and the poloidal flow remains unchanged from the equilibrium value (22). This is the physical reason for the ion mass renormalization in Eq. (21), since the mass of the plasma involved in the poloidal rotation is effectively increased due to the coupling to the parallel motion.

Quantitavely, the overall balance of the toroidal momentum in the nonlinear region can be described as follows. The perturbed toroidal velocity, $\delta V_{\zeta}=V_{\zeta}-V_{\zeta}^{(0)}$ (where $V_{\zeta}^{(0)}$ is the equilibrium toroidal velocity), is found from the radial momentum balance equation (20) and Eqs. (14), (15) and (22):

$$
\delta V_{\zeta}=-\frac{R}{n}\left(\omega-\omega_{T}\right)\left(1-\psi_{x} \frac{\partial \lambda}{\partial \psi}\right) .
$$

The net balance of toroidal momentum associated with the magnetic island motion can be written in the form

$$
m_{i} n_{0} \frac{1}{2 \pi} \frac{d}{d t} \int_{-\pi}^{\pi} d(m \hat{\theta}) \int_{r_{s}-W}^{r_{s}+W} d x \delta V_{\xi}=T_{E M}+T_{V} .
$$

Here, $T_{E M}$ is the net electromagnetic torque applied (e.g., by an external magnetic field error) to the magnetic island

$T_{E M}=\frac{1}{2} \pi \int_{-\pi}^{\pi} d(m \hat{\theta}) \int_{-\infty}^{\infty} d x \hat{\mathbf{e}}_{\xi} \cdot \mathbf{J} \times \mathbf{B}=-\frac{n c}{8 \pi R} \psi_{s}^{2} \Delta_{s}^{\prime}$.

The last term in Eq. (28) describes the flux of the toroidal momentum into the nonlinear region due to the effect of a viscosity, which can be estimated as follows:

$$
\begin{aligned}
T_{V} & =\left.\mu_{a} m_{i} n_{0} \frac{1}{2} \pi \int_{-\pi}^{\pi} d(m \hat{\theta})\left(\frac{\partial}{\partial r} \delta V_{\zeta}\right)\right|_{r_{s}-W} ^{r_{s}+W} \\
& \simeq-G_{V} \mu_{a} m_{i} n_{0}\left(\omega-\omega_{T}\right) \frac{R}{n W} .
\end{aligned}
$$

Equation (28), together with Eqs. (29) and (30), describes the evolution of the frequency of the toroidal rotation in the form

$$
\begin{aligned}
G_{W} \frac{\partial}{\partial t}\left[W\left(\omega-\omega_{T}\right)\right]= & -6 G_{V} \frac{\mu_{a}}{W}\left(\omega-\omega_{T}\right) \\
& -\frac{1}{4 \sqrt{2}}\left(\frac{r_{s}}{R}\right)^{4}\left(\frac{n S}{q}\right)^{2}\left(\frac{W}{r_{s}}\right)^{4} v_{A}^{2} \Delta_{s}^{\prime},
\end{aligned}
$$

where $G_{V}$ and $G_{W}$ are numerical coefficients, which will be estimated below.

More rigorously, Eq. (31) is derived using the second matching relation in Eq. (5). As was pointed out in Ref. 31, the imaginary part of $\Delta^{\prime}(\sin \xi$ component in our notation) describes absorption of the toroidal momentum in the nonlinear region near the resonant surface, so that Eq. (5) is equivalent to the momentum balance equation (28). Matching condition (5) requires calculation of the next order nonlinear longitudinal current $J_{\|}^{(1)}$ that is odd in $\xi$ [note that the nonlinear current in the leading order $J_{\|}^{(0)}$ given by Eq. (16) is even in $\xi$ ]. The first order current $J_{\|}^{(1)}$ responsible for absorption of the toroidal momentum in the nonlinear region is associated with viscosity effects and a slow evolution of the island width and rotation frequency. It can be determined from the same current closure equation (21) after using an orthogonality condition, which is essentially just a solvability condition for $J_{\|}^{(1)}$ obtained by surface averaging of Eq. (21). This is equivalent to the procedure of eliminating of the secular terms in an asymptotic perturbation theory. ${ }^{32}$ Then, the equation for $J_{\|}^{(1)}$ takes the form

$$
\begin{aligned}
\mathbf{B} \cdot \nabla\left(\frac{J_{\|}^{(1)}}{B}\right)-\frac{c B_{0}}{4 \pi k_{\theta} v_{a \theta}^{2}}\left[\frac { \partial } { \partial t } [ ( \omega - \omega _ { T } ) W ] \left(\frac{\partial^{2}}{\partial x^{2}} \frac{\tilde{x}}{W}\right.\right. \\
\left.-\left(\frac{\partial^{2}}{\partial x^{2}} \frac{\tilde{x}}{W}\right)\right)-\mu_{a}\left(\omega-\omega_{T}\right) W \\
\left.\quad \times\left(\frac{\partial^{4}}{\partial x^{4}} \frac{\tilde{x}}{W}-\left(\frac{\partial^{4}}{\partial x^{4}} \frac{\tilde{x}}{W}\right)\right)\right]=0,
\end{aligned}
$$

where $\tilde{x}=x-\lambda(\psi)$. Note that first order nonlinear current $J_{\|}^{(1)}$ (and thus, the amount of toroidal momentum which is transferred to the nonlinear region) is proportional to the difference between the plasma and the mode motion. ${ }^{33}$

Integrating by parts, matching condition (5) can be written in the form ${ }^{25}$

$$
\oint d \xi \int_{i}^{-\infty} d \psi \nabla_{\|} J=\frac{k_{\theta} c \Delta_{s}^{\prime}}{4 L_{S}} \tilde{\psi}^{2} .
$$

Integrating Eq. (32) over $\xi$ and $\psi$, we obtain from (33) the same equation (31), as was obtained from the semi-intuitive momentum balance (28). The numerical coefficients $G_{V}$ and $G_{W}$ in Eq. (31) can now be calculated. They are given by the relations

$$
\begin{aligned}
G_{W} & =-\frac{1}{\pi} \oint d \xi \int_{1}^{\infty} d\left(2 k^{2}\right) \frac{\langle\cos \xi\rangle}{\left(2 k^{2}-1\right)^{3 / 2}}=0.82 \\
G_{V} & =\frac{1}{\pi} \oint d \xi \int_{1}^{\infty} d\left(2 k^{2}\right)\left(4 \frac{\langle\cos \xi\rangle}{\left(2 k^{2}-1\right)^{5 / 2}}+5 \frac{\left\langle\cos ^{2} \xi\right\rangle}{\left(2 k^{2}-1\right)^{7 / 2}}\right) \\
& \simeq 2.31 .
\end{aligned}
$$


Here, $\langle\cos \xi\rangle$ and $\left\langle\cos ^{2} \xi\right\rangle$ are given by the expressions

$$
\begin{aligned}
& \langle\cos \xi\rangle=1+2 k^{2}\left(\frac{E(1 / k)}{K(1 / k)}-1\right), \\
& \left\langle\cos ^{2} \xi\right\rangle=-\frac{4}{3} k^{2}\left(2 k^{2}-1\right) \frac{E(1 / k)}{K(1 / k)}+\frac{8}{3} k^{2}\left(k^{2}-1\right)+1,
\end{aligned}
$$

where $E(1 / k)$ and $K(1 / k)$ are the elliptic integrals of the first and second kind, respectively. We have used expression (A9) for the $\lambda(\psi)$ function to calculate $G_{V}$ and $G_{W}$ in (35) and (34).

Note that the toruidal version of the current closure equation (21) with the renormalized Alfvén velocity is fully consistent with the toroidal momentum balance equation (26). Using the slab version of the current closure equation (6) instead of Eq. (32) for calculating $J_{\|}^{(1)}$, would lead to a value for the electromagnetic torque that is smaller than the one in Eq. (31) by the factor $r_{s}^{2} / q^{2} R^{2}$ and, thus, would lead to an inconsistency between Eqs. (33) and (26).

In the present work, it is assumed that the island width is sufficiently small compared to the minor plasma radius, so that the island does not affect the global plasma confinement, and the global (averaged) plasma gradients $p_{i 0}^{\prime}, T_{i 0}^{\prime}$, and $\Phi_{0}^{\prime}$ are not modified significantly by the presence of the island. Therefore, linear boundary conditions can be used for the surface functions, determining the profiles of the plasma parameters.

It can be readily seen by Eqs. (20) and (27) that the perpendicular plasma motion inside the magnetic island is locked into the island. For the case of $\omega=0$ (locked mode) the plasma velocity in the island interior is zero. In the region far outside the magnetic island $(x \gg W)$, the toroidal plasma velocity approaches its equilibrium profile. This type of profile is typically observed in the experiment ${ }^{2}$ and numerical simulations ${ }^{34,35}$ during the early stages of island growth. During the later stages of the magnetic island growth, the profiles of the radial electric field and toroidal plasma velocity become flat in the whole region; in particular, the toroidal plasma velocity in the center can be very small. Such drastic modification of the profile of the toroidal plasma velocity is probably related to the locking of plasma rotation into an $m=1$ mode, ${ }^{33,36}$ which is usually excited in the experiments, as reported in Refs. 37 and 38. The $m=1$ component can also be excited through mechanisms of nonlinear and/or toroidal coupling ${ }^{39-41}$ of different harmonics. In a number of experiments, degradation of plasma confinement ${ }^{17}$ (and hence, the evolution of mean plasma gradients) and change in the equilibrium radial electric field ${ }^{20}$ have been observed on a time scale comparable with the characteristic time scale of island growth. Such evolution of plasma parameters is related to modification of global transport in a presence of magnetic islands. ${ }^{42}$ In our simple model we neglect such effects, assuming that the profile of the toroidal velocity is modified only in the region around the magnetic island, so that $\omega_{E}$ in Eq. (14) can be considered as constant during the island evolution.

\section{Effect of neoclassical parallel viscosity on island rotation}

In this section we estimate effects of the average parallel neoclassical viscous force on mode frequency damping, which may be relevant for low aspect ratio, reactor grade tokamaks. In the tokamak magnetic configuration the toroidal viscous force vanishes due to axisymmetry. However, in the presence of a helical perturbation associated with a magnetic island, the axial symmetry is destroyed and the magnetic configuration near the rational surface becomes similar to that of stellarator, so that an additional toroidal force $F_{\zeta}=\left\langle\mathbf{B}_{\zeta} \cdot \nabla \cdot \Pi\right\rangle$ enters the momentum balance equation. The averaged neoclassical toroidal viscous force can be written in the form ${ }^{43}$

$$
\left\langle\mathbf{B}_{\zeta} \cdot \nabla \cdot \boldsymbol{\Pi}\right\rangle=3\left(\mu_{\zeta 1}^{\theta} \frac{V_{\theta}}{r}+\mu_{\zeta 1}^{\zeta} \frac{V_{\zeta}}{R}\right),
$$

where the heat flow is neglected and the viscosity coefficients in the plateau regime are given by ${ }^{43}$

$$
\begin{aligned}
& \mu_{\zeta 1}^{\theta}=\frac{\pi^{1 / 2} \Gamma(3)}{3} p_{0 i} \sum_{m, n \neq 0}\left\langle\frac{\mathbf{B}_{\zeta} \cdot \nabla B}{B} \frac{\partial b_{m n}}{\partial \theta}\right\rangle\left(\omega_{t}^{m n}\right)^{-1}, \\
& \mu_{\zeta 1}^{\hbar}=\frac{\pi^{1 / 2} \Gamma(3)}{3} p_{0 i} \sum_{m, n \neq 0}\left\langle\frac{\mathbf{B}_{\zeta} \cdot \nabla B}{B} \frac{\partial b_{m n}}{\partial \zeta}\right\rangle\left(\omega_{t}^{m n}\right)^{-1} .
\end{aligned}
$$

Here, the coefficients $b_{m n}$ are the Fourier components of the modulus of the magnetic field,

$$
B=B_{0}\left(1+\sum_{m, n} b_{m n} \cos (m \theta-n \zeta)\right)
$$

In the presence of the magnetic island, the frequency $\omega_{t}^{m n}$ does not vanish at the rational surface, and can be estimated from

$$
\omega_{t}^{m n}=v_{T i}\left|\frac{m-n q}{q R}\right| \simeq \frac{k_{\theta} W}{L_{S}} v_{T i} .
$$

The modulus of the magnetic field in the vicinity of the rational surface is

$$
B \simeq B_{0}\left(1+\tilde{B}_{\theta} \frac{B_{\theta}}{B_{0}^{2}}\right),
$$

where $B_{\theta}$ is the equilibrium poloidal magnetic field and $\widetilde{B}_{\theta}$ is the perturbation due to an island whose order of magnitude can be estimated as

$$
\tilde{B}_{\theta}=\frac{\partial \tilde{\psi}}{\partial r} \simeq \frac{\tilde{\psi}}{W} \simeq B_{0} \frac{W}{L_{S}} .
$$

Then, the modulus of the magnetic field in the presence of perturbation with helicity $(m, n)$ can be written in a form similar to the stellarator field,

$$
B=B_{0}\left[1-\epsilon \cos \theta+\epsilon_{W} \cos (m \theta-n \zeta)\right],
$$

where 


$$
\epsilon_{W}=\alpha_{W} \frac{r_{s}}{R} \frac{W}{q L_{S}} .
$$

and $\epsilon_{W}$ is the numerical coefficients of the order of unity.

Using Eq. (40), the relevant viscosity coefficients are given approximately by

$$
\begin{aligned}
& \mu_{\zeta}^{\theta} \simeq-\frac{\pi^{1 / 2} \Gamma(3)}{6} \frac{p_{0 i}}{\omega_{t}^{m n}} \frac{n m \epsilon_{W}^{2} B_{0}}{R}, \\
& \mu_{\zeta}^{\zeta} \simeq \frac{\pi^{1 / 2} \Gamma(3)}{3} \frac{p_{0 i}}{\omega_{t}^{m n}} \frac{n^{2} \epsilon_{W}^{2} B_{0}}{R},
\end{aligned}
$$

and the neoclassical toroidal viscous force is

$$
\left\langle\mathbf{B}_{\zeta} \cdot \nabla \cdot \boldsymbol{\Pi}\right\rangle=\frac{\pi^{1 / 2} \Gamma(3)}{2} p_{0 i} \frac{n \epsilon_{w}^{2} B_{0}}{\omega_{t} R}\left(-\frac{m V_{\theta}}{r}+\frac{n V_{t}}{R}\right)
$$

Then, the damping of the island rotation due to the neoclassical viscosity is described by the equation

$$
\frac{d}{d t}\left(\omega-\omega_{T}\right) \simeq-\tau_{t}^{-1}\left(\omega-\omega_{T}\right)
$$

with the rate given by

$$
\tau_{t}^{-1} \simeq \frac{v_{T i}^{2} \epsilon_{W}^{2} n^{2}}{R^{2} \omega_{t}^{m n}} \simeq \frac{v_{T i}}{q R} \frac{n S}{q^{3}}\left(\frac{r_{s}}{R}\right)^{4} \frac{W}{r_{s}} .
$$

In this work we limit ourselves to a quantitative estimation of the effect of the damping of the island rotation due to a neoclassical viscous force in the toroidal direction. This slowing down effect is indeed negligible for medium-sized, large aspect ratio machines, but may be relevant for a Joint European Torus $^{44}$ (JET) sized tokamak with reactor grade temperatures $\left(T_{i} \approx 5-10 \times 10^{3} \mathrm{eV}\right)$ at the $q=2$ surface, when the rotation damping rate could be comparable with other time scales typical of tearing instabilities.

\section{STRUCTURE OF THE MAGNETIC PERTURBATION IN THE LINEAR REGION}

In the external (to the magnetic island) or linear region the tearing mode perturbation is described by the ideal MHD equations. In the large aspect ratio tokamak ordering and neglecting toroidal mode coupling, the magnetic field perturbation can be represented by the helical flux function,

$$
\Psi[r, \hat{\theta}, \phi(t), t]=\psi(r, t) \cos [m \hat{\theta}-\phi(t)],
$$

where $\phi(t)$ is the phase of the perturbation.

Referring to Fig. 1, we consider a plasma with circular cross section of radius $a$, surrounded by a coaxial resistive wall of radius $d$ and thickness $\delta$. To model the magnetic field created by external coils, we assume a helical surface current $I(t)=I_{0} \cos \left[m \hat{\theta}-\phi_{I}(t)\right]$ with the same helicity ratio $\mathrm{m} / \mathrm{n}$ located at the radius $r=b, a<b<d$. The magnetic field created by this current is resonant at the rational surface $r=r_{s}$ with the safety factor $q_{s}=m / n$. The plasma region is divided in two parts: $A$ for $0<r<r_{s}$ and $B$ for $r_{s}<r<a$. Vacuum region $V$ is located between $a$ and $d$. The wall region is labeled $M$, and the region $r>d+\delta$ is outside the

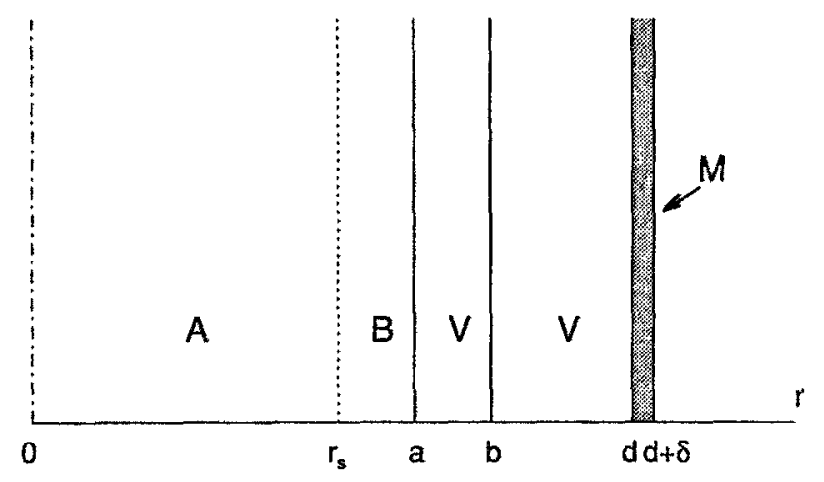

FIG. I. The regions where the helical flux function $\Psi$ is determined within the linear approximation: $A$ and $B$ - plasma regions $\left(r=r_{s}\right.$ is the location of the rational surface, $a$ is the minor plasma radius); $V$ - vacuum region; the external coils are located at $r=b ; M$ is the metal wall of the thickness $\delta$.

vacuum chamber. The magnetic flux in the linear region is described by the equation for an ideal equilibrium with an external driving term, ${ }^{45}$

$$
\nabla_{ \pm}^{2} \Psi-\frac{J_{0 z}^{\prime}}{B_{\theta}[1-(n / m) q(r)]} \Psi=\frac{4 \pi}{c} I(t) \frac{\delta(r-b)}{b} .
$$

The solution of this equation with the boundary conditions at different interfaces is straightforward, and for some particular cases has been presented in Refs. 8, 46, and 47, assuming stationary rotation with constant frequency. In this section we generalize the previous results for an arbitrary time dependence in the magnetic island and external current, so that the obtained relations can be used in modeling of the dynamical behaviour of tearing modes with an applied resonant magnetic perturbation, which is important, for instance, for the problem of feedback stabilization of magnetic islands. We eliminate also the so-called "tenuous" plasma approximation ${ }^{46}$ and take into account an effect of a finite plasma current in the region $r_{s}<r<a$.

In region $A$ the regularity at the origin requires that the solution of the second order equation (51) be of the type $\left(F_{0} \rightarrow r^{m}\right.$ as $\left.r \rightarrow 0\right)$

$$
\Psi_{A}(r)=C_{A} F_{0}(r)
$$

while in region $B$ the solution $\Psi_{B}(r)$ can be expressed as a linear combination of two linearly independent eigenfunctions $F_{+}(r)$ and $F_{-}(r)$,

$$
\Psi_{B}(r)=C_{B}^{+} F_{+}(r)+C_{B}^{-} F_{-}(r),
$$

with the boundary conditions

$$
F_{+}^{\prime}(a)-\frac{m}{a} F_{+}(a)=0, \quad F_{-}^{\prime}(a)+\frac{m}{a} F_{-}(a)=0 .
$$

For a class of generalized parabolic current profiles the solution of Eq. (51) can be obtained analytically, ${ }^{48}$ so that $F_{+}(r)$, and $F_{-}(r)$ can be calculated explicitly. In general, they require numerical evaluation. After matching $\Psi$ at the different interfaces we obtain for the total magnetic flux in the plasma region $B$, 


$$
\begin{aligned}
\Psi_{B}(r, t)= & \psi_{s}(t) F_{-}(r)+\frac{2 \pi}{m c} I(t)\left(\frac{a}{b}\right)^{m} \frac{1}{F_{+}(a)}\left[F_{-}(r)\right. \\
& \left.-F_{+}(r)\right]+\left(\frac{a}{d}\right)^{m} \frac{1}{f_{a, d}} \frac{1}{F_{+}(a)} \\
& \times[g(t)-\hat{D} g(t)]\left[F_{-}(r)-F_{+}(r)\right] .
\end{aligned}
$$

Here, $\psi_{s}(t) \equiv \Psi_{A}\left(r_{s}, t\right)=\Psi_{B}\left(r_{s}, t\right)$ is the value of reconnected flux at the rational surface, function $g(t)$ is defined by the relation

$$
g(t)=\psi_{s}(t)\left(\frac{a}{d}\right)^{m} F_{-}(a)-\frac{2 \pi}{m c} I(t)\left(\frac{b}{d}\right)^{m} f_{a, b},
$$

and parameters $f_{a, b}$ and $f_{a, d}$ are given by

$$
f_{a, r}=1-\left(\frac{a}{r}\right)^{2 m} \frac{F_{-}(a)}{F_{+}(a)} \text {. }
$$

The time delay (due to the resistive wall) operator $\hat{D}$ is defined by the expression

$$
\hat{D} g(t)=\frac{1}{\tau_{w}} \int_{t_{0}}^{t} g\left(t^{\prime}\right) \exp \left(\frac{t^{\prime}-t}{\tau_{w}}\right) d t^{\prime},
$$

with the effective wall constant $\tau_{w}=\tau_{R} f_{a, d}$, where $\tau_{R}=\delta d / 2 m D_{0}, D_{0}=c^{2} / 4 \pi \sigma$ is the magnetic diffusivity, and $\sigma$ is the conductivity of the metal wall. The constant $\Psi$ approximation has been employed for the thin resistive shell. ${ }^{47}$ The normalization $F_{0}\left(r_{s}\right)=F_{-}\left(r_{s}\right)=F_{+}\left(r_{s}\right)=1$ is used.

Note that the magnetic flux function (55) is a solution of the MHD equilibrium equation in neglect of toroidal effects and effects of plasma pressure. In this approximation, it consists of three principal parts. The first part is associated with the final value of the reconnected flux at the rational surface and proportional to $\psi_{s}(t)$. This part corresponds to a no wall eigenmode, which decays asymptotically at infinity. The second part is driven by the external perturbation and is proportional to the current in the external coils $I(t)$. The third part describes the perturbation "reflected" from the wall, and can be considered as part of the magnetic flux created by the image currents in the resistive wall. These image currents are induced by the plasma current at the rational surface and the current in the external coils $I(t)$. The second and third terms in Eq. (55) represent what is often called the "penetrated" flux, since this part of the flux is created by the currents outside the plasma. It is apparent that the "penetrated" flux vanishes exactly at the rational surface $r=r_{s}$, regardless of the form of the functions $F_{+}(r), F_{-}(r)$. The penetrated flux is identically zero for $r<r_{s}$ because within the linear boundary matching problem the solution $\psi_{A}(r)$ in region A does not respond to the boundary conditions at $r=a$ as long as the magnetic flux is fixed at the rational surface. This represents the so-called "shielding effect" of the singular layer at $r=r_{s}$, which is merely a property of the solution of an ordinary second-order differential equation. The effect of $I(t)$ on the value of the magnetic flux at the rational surface (and, hence, on the magnetic island width) appears only in the nonlinear magnetic island evolution through the external boundarv condition for the nonlinear region. These boundary conditions are ultimately determined by the asymptotic behaviour of the flux function (55) in region $\mathrm{B}$, as $r \rightarrow r_{s}$.

Thus, the boundary value problem considered in this section is now cast into a form that exhibits the superposition of effects due to the plasma and external sources. The magnitude and phase of the magnetic flux perturbation reflected from the conducting wall is determined by the wall position and its resistivity. Then, there are only two components in $\Psi_{B}(r, t)$ and these are linearly independent. One is induced by the plasma current at the rational surface, and is thus proportional to the reconnected flux, and the other is caused by the external magnetic perturbation. In general, these two components of $\Psi_{B}(r, t)$ have different time dependences (in particular, different frequencies). The finite wall resistivity introduces a finite time delay (described by operator $\hat{D}$ ) in the plasma response. Clearly, the driven reconnecting flux is neither strictly proportional to nor in phase with the applied magnetic field. This should be taken into account in sorting out the measured signals and in devising a feedback loop.

A perfectly conducting wall does not introduce any time delay $(\hat{D}=0)$, in which case the expression for the magnetic flux takes the form

$$
\begin{aligned}
\Psi_{B}(r, t)= & \frac{\psi_{s}(t)}{f_{a, d}}\left[F_{-}(r)-\frac{F_{-}(a)}{F_{+}(a)}\left(\frac{a}{d}\right)^{2 m} F_{+}(r)\right]+\frac{2 \pi}{m c} I(t) \\
& \times\left(\frac{a}{b}\right)^{m} \frac{1-(b / d)^{2 m}}{F_{+}(a)}\left[F_{-}(r)-F_{+}(r)\right]
\end{aligned}
$$

If the wall is removed ( $d \rightarrow \infty$ or $\tau_{w} \rightarrow 0$, that is $\hat{D}=1$ ) the reflected flux disappears:

$$
\begin{aligned}
& \Psi_{B}(r, t)=\psi_{s}(t) F_{-}(r)+\Psi^{e x t}(r, t), \\
& \Psi^{e x t}(r, t)=\frac{2 \pi}{m c} I(t)\left(\frac{a}{b}\right)^{m} \frac{1}{F_{+}(a)}\left[F_{-}(r)-F_{+}(r)\right] .
\end{aligned}
$$

Usually, the magnetic field perturbation is calibrated with respect to the the signal registered without plasma. In the latter case, the magnetic flux perturbation is described by the vacuum expression

$$
\Psi_{V}=-\frac{2 \pi}{m c}\left[I(t)-\left(\frac{b}{d}\right)^{2 m}(I(t)-\hat{D} I(t))\right]\left(\frac{r}{b}\right)^{m} .
$$

Often the plasma region B is described in terms of the "tenuous plasma" (vacuum) approximation, ${ }^{46}$ so that the vacuum solution is used for $\Psi_{B}$. In the present model the functions $F_{+}, F_{-}$describe the effect of finite plasma current in region $\mathrm{B}$, which cannot be neglected in general.

To perform matching between the linear and nonlinear regions, two components of the $\Delta^{\prime}$ parameter are required in Eqs. (4) and (5). These matching parameters are defined by the relations

$$
\Delta_{c}^{\prime}=\frac{1}{\pi \psi_{s}} \int_{-\pi}^{\pi}\left[\Psi_{B}^{\prime}(r)-\Psi_{A}^{\prime}(r)\right] \cos [m \hat{\theta}-\phi(t)] d(m \hat{\theta}),
$$




$$
\Delta_{s}^{\prime}=\frac{1}{\pi \psi_{s}} \int_{-\pi}^{\pi}\left[\Psi_{B}^{\prime}(r)-\Psi_{A}^{\prime}(r)\right] \sin [m \hat{\theta}-\phi(t)] d(m \hat{\theta}) .
$$

We represent the linear tearing-mode parameter $\Delta^{\prime}$ as a sum of the mode induced and driven part,

$$
\Delta_{c, s}^{\prime}=\Delta_{(c, s), \text { mode }}^{\prime}+\Delta_{(c, s), \text { coil }}^{\prime} .
$$

The mode induced part can be written in the form

$$
\begin{aligned}
\Delta_{c, \text { mode }}^{\prime}= & \Delta_{-}^{\prime}+\left(\frac{a}{d}\right)^{2 m} \frac{F_{-}(a)}{F_{+}(a)} \frac{1}{f_{a, d}}\left(\Delta_{-}^{\prime}-\Delta_{+}^{\prime}\right) \\
& \times\left(1-\cos \Delta \Phi^{R}\right), \\
\Delta_{s, \text { mode }}^{\prime}= & -\left(\frac{a}{d}\right)^{2 m} \frac{F_{-}(a)}{F_{+}(a)} \frac{1}{f_{a, d}}\left(\Delta_{-}^{\prime}-\Delta_{+}^{\prime}\right) \sin \Delta \Phi^{R} .
\end{aligned}
$$

Here, $\Delta \Phi^{R}(t)$ is the phase shift in the plasma response introduced by the resistive wall,

$$
\begin{aligned}
\cos \Delta \Phi^{R}= & \frac{1}{\tau_{w}} \int_{\tau_{0}}^{t} \exp \left(-\frac{t-t^{\prime}}{\tau_{w}}\right) \frac{\tilde{\psi}\left(t^{\prime}\right)}{\psi(t)} \\
& \times \cos \left[\phi(t)-\phi\left(t^{\prime}\right)\right] d t^{\prime}, \\
\sin \Delta \Phi^{R}= & \frac{1}{\tau_{w}} \int_{t_{0}}^{t} \exp \left(-\frac{t-t^{\prime}}{\tau_{R}}\right) \frac{\tilde{\psi}\left(t^{\prime}\right)}{\psi(t)} \\
& \times \sin \left[\phi(t)-\phi\left(t^{\prime}\right)\right] d t^{\prime} .
\end{aligned}
$$

The parameters $\Delta_{ \pm}^{\prime}$ are defined by relations

$$
\begin{aligned}
& \Delta_{+}^{\prime}=\left.\frac{d}{d r}\left[F_{+}(r)\right]\right|_{r \rightarrow r_{s}+0}-\left.\frac{d}{d r}\left[F_{0}(r)\right]\right|_{r \rightarrow r_{s}-0}, \\
& \Delta_{-}^{\prime}=\left.\frac{d}{d r}\left[F_{-}(r)\right]\right|_{r \rightarrow r_{s}+0}-\left.\frac{d}{d r}\left[F_{0}(r)\right]\right|_{r \rightarrow r_{s} \sim 0} .
\end{aligned}
$$

The externally driven ${ }^{49}$ part of the tearing-mode stability index is given by the expressions

$$
\begin{aligned}
\Delta_{c, \text { coil }}^{\prime}= & \frac{2 \pi}{m c} \frac{I_{0}(t)}{\tilde{\psi}(t)}\left(\frac{a}{b}\right)^{m} \frac{1}{F_{+}(a) f_{a, d}}\left[1-\left(\frac{b}{d}\right)^{2 m}\right]\left(\Delta_{-}^{\prime}-\Delta_{+}^{\prime}\right) \\
& \times \cos \Delta \Phi(t)+\frac{2 \pi}{m c} \frac{I_{0}(t)}{\tilde{\psi}(t)} \frac{(a b)^{m}}{d^{2 m}} \frac{1}{F_{+}(a)} \\
& \times \frac{f_{a, b}}{f_{a, d}}\left(\Delta_{-}^{\prime}-\Delta_{+}^{\prime}\right) \cos \left[\Delta \Phi(t)+\Delta \Phi_{l}^{R}\right], \\
\Delta_{s, \text { coils }}^{\prime}= & \frac{2 \pi}{m c} \frac{I_{0}(t)}{\tilde{\psi}(t)}\left(\frac{a}{b}\right)^{m} \frac{1}{F_{+}(a) f_{a, d}}\left[1-\left(\frac{b}{d}\right)^{2 m}\right]\left(\Delta_{-}^{\prime}-\Delta_{+}^{\prime}\right) \\
& \times \sin \Delta \Phi(t)+\frac{2 \pi}{m c} \frac{I_{0}(t)}{\tilde{\psi}(t)} \frac{(a b)^{m}}{d^{2 m}} \frac{1}{F_{+}(a)} \frac{f_{a, b}}{f_{a, d}} \\
& \times\left(\Delta_{-}^{\prime}-\Delta_{+}^{\prime}\right) \sin \left[\Delta \Phi(t)+\Delta \Phi_{I}^{R}\right],
\end{aligned}
$$

where $\Delta \Phi(t)$ is the phase shift between the applied external current and the magnetic island:

$$
\Delta \Phi(t)=\phi(t)-\phi_{l}(t) .
$$

The phase delay $\Delta \Phi_{I}^{R}$ in the externally driven part of $\Delta^{\prime}$ due to the wall resistivity is introduced by the following relations;

$$
\begin{aligned}
\cos \left[\Delta \Phi(t)+\Delta \Phi_{I}^{R}\right]= & \frac{1}{\tau_{w}} \int_{t_{0}}^{t} \exp \left(-\frac{t-t^{\prime}}{\tau_{w}}\right) \frac{I_{0}\left(t^{\prime}\right)}{I_{0}(t)} \\
& \times \cos \left[\phi(t)-\phi_{l}\left(t^{\prime}\right)\right] d t^{\prime}, \\
\sin \left[\Delta \Phi(t)+\Delta \Phi_{I}^{R}\right]= & \frac{1}{\tau_{w}} \int_{t_{0}}^{t} \exp \left(-\frac{t-t^{\prime}}{\tau_{w}}\right) \frac{I_{0}\left(t^{\prime}\right)}{I_{0}(t)} \\
& \times \sin \left[\phi(t)-\phi_{I}\left(t^{\prime}\right)\right] d t^{\prime} .
\end{aligned}
$$

Expressions (65)-(76) are written for the general case of an arbitrary time dependence in the external current and the magnetic island width. In this form the time-dependent parameters $\Delta_{(c, s)}^{\prime}$ should be calculated numerically. They may be useful in the implementation of a feedback stabilization loop.

For analytic estimates we use steady-state approximation, assuming the quasistationary island rotation with a frequency much larger than the inverse time scale for the evolution of the island width, i.e., $\omega \gg(1 / W)(\partial W / \partial t)$. The current in the external coils is assumed to be rotating with the frequency $\omega_{1}$ and a constant amplitude $I_{0}$. Then, the wall induced phase delay can be reduced to the form

$$
\begin{aligned}
& \tan \Delta \Phi^{R}=\omega \tau_{w}, \\
& \tan \Delta \Phi_{l}^{R}=\omega_{l} \tau_{w} .
\end{aligned}
$$

In this form the phase delay introduced by the resistive wall has been previously obtained in Ref. 10 for the case $\omega=\omega_{1}$.

The second term in expression (66) describes the influence of the wall on the stability of a rotating mode, with the term $\cos \Delta \Phi^{R}$ parametrizing the effect of the wall resistivity. For a perfectly conducting wall $\left(\omega \tau_{w} \gg 1\right)$, the reflected flux is out of phase with the magnetic island, and $\Delta \Phi^{R}=\pi / 2$, which corresponds to "perfect mirror" reflection from the wall. Note that the effect of the wall is sensitive to the profile of the plasma current only in the region $r_{s}<r<a$. The plasma current inside the rational surface $\left(0<r<r_{s}\right)$ does not affect reflected fiux, since the conbination $\Delta_{-}^{\prime}-\Delta_{+}^{\prime}$ does not depend on the current profile in the region $0<r<r_{s}$. In the tenuous plasma approximation, the functions $F_{-}(r)$ and $F_{-}(r)$ can be approximated by the vacuum form, $F_{ \pm}(r)=\left(r / r_{s}\right) \pm m$. Then, expression (66) takes the form

$$
\begin{aligned}
\Delta_{c, \text { made }}^{\prime}= & \Delta_{-}^{\prime}-\frac{2 m}{r_{s}}\left(r_{s} d\right)^{2 m}\left[1-\left(\frac{r_{s}}{d}\right)^{2 m}\right]^{-1} \\
& \times\left(1-\cos \Delta \Phi^{R}\right),
\end{aligned}
$$

In the tenuous plasma approximation the conducting wall is stabilizing for rotating magnetic islands ${ }^{10,47,50,51}$ [the second term in (79) is always negative, which reduces the total $\Delta_{c}^{t}$ ]. In general, a wall of finite conductivity stabilizes the mode growth under the condition $\Delta_{-}^{\prime}-\Delta_{+}^{\prime}<0$, which normally holds for a typical current profile. (Note that the conducting wall is destabilizing for a current profile which produces $\Delta_{-}^{\prime}-\Delta_{+}^{\prime}>0$.) For a slowly rotating mode 
$\left(\omega \tau_{w} \ll 1\right)$ the effect of the wall disappears and an effect of the external field is maximal for $\Delta \Phi=0$ (it is stabilizing, assuming $\Delta_{-}^{\prime}-\Delta_{+}^{\prime}<0$ ). In the general case, $\omega \tau_{w} \simeq 1$, the magnetic flux reflected from the resistive wall [given by the second term in Eq. (72)], has a finite phase shift with respect to the part directly induced by the external current [the latter is described by the first term in Eq. (72)]. Thus, the conducting wall may decrease the effectiveness of the applied external field in the mode stabilization due to interference between these two components. If one chooses to stabilize the rotating mode by applying the external magnetic field rotating at the same frequency as the mode $\left(\omega_{1}=\omega\right)$, a finite phase shift $\Delta \Phi=-\delta$ has to be maintained between the mode and the external current to maximize the stabilization effect. The value of the phase shift depends on the wall resistivity and can be determined from equation (72):

$$
\cot \delta=\cot \Delta \Phi_{I}^{R}+\frac{1}{\sin \Delta \Phi_{I}^{k}} \frac{1-(b / d)^{2 m}}{(b / d)^{2 m} f_{a, b}} .
$$

This phase shift (which is in general of the order of $\Delta \Phi_{I}^{R}$ ) should be taken into account in designing of feedback stabilization experiments.

\section{NONLINEAR DYNAMICS OF THE MAGNETIC ISLANDS}

\section{A. Slowing down of the island rotation in the Rutherford regime}

In this section we consider the simplest case of magnetic island dynamics in the absence of an external drive and interaction with the wall. We assume that the magnetic island appears because the profile of the plasma current is such that $\Delta_{c}^{\prime}>0$. For a sufficiently large island width, the effects of the perturbed bootstrap current, plasma inertia and viscosity can be neglected, and the island growth is described by the Rutherford equation ${ }^{9}$

$$
G_{1} \frac{\partial W}{\partial t}=D_{R} \frac{\Delta_{c}^{\prime}}{4} .
$$

In this regime the magnetic island width grows linearly with time,

$$
W(t)=W(0)+\Delta_{c}^{\prime} r_{s} t / \tau_{D},
$$

where $\tau_{D}=4 G_{1} r_{s}^{2} / D_{R}$ and $W \rightarrow W / r_{s}$. With the above approximations the evolution of the frequency of island rotation is described simply by the equation

$$
\frac{\partial}{\partial t}\left[W\left(\omega-\omega_{T}\right)\right]=0 .
$$

This equation means that the toroidal plasma momentum is conserved in the magnetic island in the absence of external torques. As the island grows in width, more plasma mass is involved in the rotation. Thus, for the constant toroidal momentum the frequency of island rotation decreases, and it is described by the equation

$$
\omega=\omega_{T}+\frac{\text { const }}{W(t)}=\omega_{T}+\frac{\text { const }}{W(0)+\Delta_{c}^{\prime} r_{s} t / \tau_{D}} .
$$

This equations obtained assuming that the island width grows on a time scale faster than characteristic time scale of the anomalous viscous damping, that is, a radial transfer of the toroidal momentum from the island region to the bulk plasma associated with an anomalous viscosity is negligible. The effect of viscous drag modifies the evolution of the rotation:

$$
\omega=\omega_{T}+\frac{\text { const }}{W(t)} \exp \left(-\frac{\tau_{D}}{\tau_{M}} \frac{1}{\Delta_{c}^{\prime} r_{s}} \frac{1}{W(t)}\right),
$$

where $\tau_{M}=\left(G_{W} / 6 G_{V}\right) r_{s}^{2} / \mu_{a}$ is the rate of anomalous momentum damping. In our model we assume that during the evolution of a magnetic island the toroidal velocity follows to "adiabatic" profiles of the electric field, ion pressure and poloidal velocity according to equation (20). These "adiabatic" profiles are essentially obtained, neglecting the anomalous viscosity effects. We suppose that the momentum balance in the radial direction (20) is established on a much faster Alfvénic time scale.

The equation (85) essentially means that in the absence of external torque the island rotation is damped to the frequency of the equilibrium rotation of the bulk plasma, which is essentially determined by the radial momentum balance. ${ }^{52}$ In the approximation $\tau_{M} \gg \tau_{D}$, the damping rate is mainly determined by the growth rate of the island width in the Rutherford regime. This appears to be consistent with the experimental observations made on the Texas Experimental Tokamak ${ }^{20,53}$ (TEXT).

A stationary magnetic structure of the type (1) moving across the magnetic field lines with the frequency $\omega_{\mathcal{T}}$ can be registered by an array of external magnetic probes as rotating both poloidally and toroidally. The corresponding frequencies are defined from the condition $\xi=m\left(\theta-\zeta / q_{s}\right)-\omega_{T} t=$ const $:$

$$
\begin{aligned}
& \frac{d \zeta}{d t}=-\frac{\omega_{T}}{n}=-\frac{1}{n}\left(\omega_{E}+\omega_{* p}+k \omega_{* T}\right), \\
& \frac{d \theta}{d t}=\frac{\omega_{T}}{m}=\frac{1}{m}\left(\omega_{E}+\omega_{* p}+k \omega_{* T}\right) .
\end{aligned}
$$

Note that the poloidal mode rotation with the frequency $\omega_{T} / m$ does not contradict equation (22) for the poloidal plasma rotation. The poloidal mode rotation does not necessarily mean that the plasma moves in the poloidal direction with the same frequency because there can be free plasma streaming along the magnetic field lines. In fact, as mentioned in Section IV B, the poloidal component of plasma motion along the magnetic field partially compensates the poloidal component of the transverse flow, so that the net plasma poloidal velocity is defined just by the temperature gradient, as indicated in Eq. (22).

\section{B. Bifurcations into an island-type equilibrium and the critical magnetic field for locked modes}

In this section we consider the case of initially stable tearing modes $\left(\Delta_{c}^{\prime}<0\right)$, so that a magnetic island can appear only due to some destabilizing effect, e.g., bootstrap current, plasma inertia, viscosity and/or an external drive. The dy- 
namics of bootstrap current driven islands has been considered in Ref. 30. Thus, we neglect this effect here for simplicity. The full nonlinear dynamics of the magnetic island is described by the following nonlinear evolution equations;

$$
\begin{aligned}
& \tau_{D} \frac{\partial}{\partial t} W=r_{s} \Delta_{c}^{\prime}-\frac{T_{A}^{2}}{W^{3}}\left(\Omega-\Omega_{E}\right)(\Omega-1), \\
& \tau_{M} \frac{\partial}{\partial t}[W(\Omega-1)]=-\frac{\Omega-1}{W}-\frac{T_{M}}{T_{A}^{2}} r_{s} \Delta_{s}^{\prime} W^{4} .
\end{aligned}
$$

Here,

$$
\begin{aligned}
& T_{A}^{2}=4 G_{3}\left(\frac{q}{n S}\right)^{2}\left(\frac{R}{r_{s}}\right)^{2} \tau_{A}^{2} \omega_{T}^{2}, \\
& T_{M}=\frac{G_{3}}{\sqrt{2} G_{W}} \tau_{M} \omega_{T},
\end{aligned}
$$

where $\tau_{A}=R / v_{A}$ is the characteristic Alfvén time, and the frequency is normalized to the toroidal rotation frequency, $\Omega \equiv \omega / \omega_{T}$.

We consider equilibrium magnetic islands excited by a static external perturbations, i.e., $\omega_{I}=0$. Such perturbations can be produced, for instance, by residual etror fields. For these external perturbations the effect of the wall resistivity disappears and the perturbed magnetic flux can be described by expression (60), which corresponds to the case of no wall. We assume that the magnetic island driven by a static external perturbation does not rotate either, i.e., $\omega=0$. Then the corresponding $\Delta^{\prime}$ parameters can be written in the form

$$
\begin{aligned}
& \Delta_{c}^{\prime}=\Delta_{-}^{\prime}+\frac{W_{v}^{2}}{W^{2}}\left(\frac{a}{r_{s}}\right)^{m} \frac{1}{F_{+}(a)}\left(\Delta_{-}^{\prime}-\Delta_{+}^{\prime}\right) \cos \Delta \Phi, \\
& \Delta_{s}^{\prime}=\frac{W_{v}^{2}}{W^{2}}\left(\frac{a}{r_{s}}\right)^{m} \frac{1}{F_{+}(a)}\left(\Delta_{-}^{\prime}-\Delta_{+}^{\prime}\right) \sin \Delta \Phi .
\end{aligned}
$$

Here, the parameter $W_{v}$ is defined by

$$
W_{v}^{2}=\frac{4 L_{S}}{B_{0}} \frac{2 \pi}{m c} I_{0}\left(\frac{r_{s}}{b}\right)^{m},
$$

and has a meaning of an equivalent magnetic island at $r=r_{s}$ obtained by superimposing the vacuum magnetic field (62) onto the equilibrium.

In the absence of stabilizing effects due to plasma rotation, any applied resonant magnetic perturbation leads to the appearance of a finite magnetic island with the width defined by the condition $\Delta_{c}^{\prime}=0$ :

$$
W^{2}=\Gamma^{2} W_{v}^{2} .
$$

The factor $\Gamma$ describes amplification of the island due to the plasma response: ${ }^{54,55}$

$$
\Gamma^{2}=\left(\frac{a}{r_{s}}\right)^{m} \frac{1}{F_{+}(a)}\left(1-\frac{\Delta_{+}^{\prime}}{\Delta_{-}^{\prime}}\right)
$$

Amplification of the magnetic island width depends upon the profile of the plasma current and mostly defined by the stability index $\Delta_{-}^{\prime}$. Under the conditions $\Delta_{-}^{\prime}-\Delta_{+}^{\prime}<0$ and
$\Delta_{-}^{\prime}<0$, the $O$ point of the magnetic island is located at the minimum of the extemal current, which corresponds to the phase shift $\Delta \Phi=\pi$.

The appearance of the magnetic island at the rational surface causes a change in the radial magnetic field measured at the plasma boundary $r=a$, which is given by

$$
\frac{\left|\tilde{B}_{r}\right|}{\left|\tilde{B}_{r}^{v}\right|}=\left|1-\frac{F_{-}(a)}{F_{+}(a)} \frac{\Delta_{+}^{\prime}}{\Delta_{-}^{\prime}}\right|,
$$

where $\tilde{B}_{r}$ and $\tilde{B}_{r}^{v}$ are given by

$$
\tilde{B}_{r}=-\left.\frac{1}{a} \frac{\partial}{\partial \theta} \Psi_{B}\right|_{r=a}, \quad \tilde{B}_{r}^{v}=-\left.\frac{1}{a} \frac{\partial}{\partial \theta} \Psi_{V}\right|_{r=a} .
$$

Note that $\tilde{B}_{r}$ contains both the components due to the external field and that created by the magnetic island [see Eq. (55) for the total magnetic flux]; $\tilde{B}_{r}$ is the total magnetic field at the plasma boundary in the presence of the magnetic island induced by an external current $I_{0}$, and $\tilde{B}_{r}^{v}$ is the amplitude of the magnetic field produced in vacuo by the external current of the same amplitude. One must remember that relations (95), (96) and (97) are applicable only to the case of magnetic islands in the absence of any stabilizing effects of plasma rotation and viscosity.

Now we consider bifurcation of a magnetic equilibrium into the state with a magnetic island of finite width caused by the external magnetic field and taking into account the effects of the plasma inertia and viscosity. We assume that the induced magnetic island is in a stationary state with $W=$ const, and $\omega=0$. The basic nonlinear equations for such an equilibrium can be written in the following form:

$$
\begin{aligned}
& r_{s} \Delta_{-}^{\prime}+\Gamma^{2} \frac{W_{v}^{2}}{W^{2}} r_{s} \Delta_{-}^{\prime} \cos \Delta \Phi-\frac{T_{A}^{2}}{W^{3}}=0, \\
& \frac{1}{W}-\frac{T_{M}}{T_{A}^{2}} \Gamma^{2} W_{v}^{2} W^{2} r_{s} \Delta_{-}^{\prime} \sin \Delta \Phi=0 .
\end{aligned}
$$

For simplicity we have assumed in Eqs. (99) and (100) that $\omega_{E} \gg\left(\omega_{* p}, \omega_{* T}\right), \Omega_{E} \simeq 1$, which is typically satisfied for tokamak discharges before application of an external field.

The first term in Eq. (99) is the standard Rutherford term (which is stabilizing here); the second term describes destabilization due to an external drive $\left(\Gamma^{2} W_{v}^{2}\right.$ is proportional to the magnitude of an external current), and the last term is due to the stabilizing inertia effect.

It is convenient to introduce a new variable $g$, which is related to the magnetic island width by the relation

$$
W^{3}=g^{3} \frac{T_{A}^{2}}{-2 r_{s} \Delta_{-}^{\prime}} .
$$

Eliminating the phase shift $\Delta \Phi$ from Eqs. (99) and (100), we obtain for the stationary state,

$$
\begin{aligned}
\Gamma^{4} W_{v}^{4} & =\frac{4}{g^{6} T_{M}^{2}}+\left(\frac{T_{A}^{2}}{-2 r_{s} \Delta_{-}^{\prime}}\right)^{4 / 3} g^{4}\left(\frac{2}{g^{3}}+1\right)^{2} \\
& =\left(\frac{T_{A}^{2}}{-2 r_{s} \Delta_{-}^{1}}\right)^{4 / 3} P(g, \zeta) .
\end{aligned}
$$




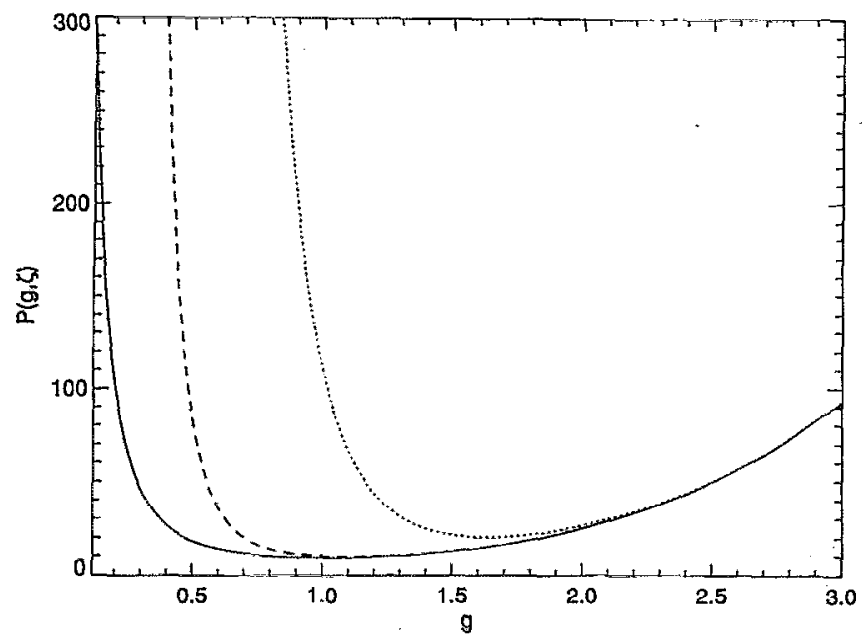

FIG. 2. The function $P(g, \zeta)$ versus the normalized island width $g$ for different values of the parameter $\zeta$, which is the normalized viscosity coefficient $\zeta ; \zeta=0$ (solid line), $\zeta=1$ (dashed line), $\zeta=5$ (dotted line). The minumum of the function $P(g, \zeta)$ determines the critical amplitude of the external error field required to induce the magnetic island.

The function $P(g, \zeta)$ is defined by the relation

$$
P(g, \zeta)=\frac{4}{g^{2}}+4 g+g^{4}+\frac{\zeta^{2}}{g^{6}},
$$

and the parameter $\zeta$ characterizing the effect of an anomalous plasma viscosity is given by

$$
\zeta=\frac{2\left(-2 r_{s} \Delta_{-}^{\prime}\right)^{2 / 3}}{T_{M} T_{A}^{4 / 3}} .
$$

A plot of $P(g, \zeta)$ as a function of $g$ for different values of the parameter $\zeta$ is shown in Fig. 2. For the external magnetic field larger than some critical value, there are two solutions of the equation for stationary magnetic islands (102): one is stable and another one is unstable with respect to small deviations from the stationary. As the magnitude of the external current reduces, the two stationary points become closer and they merge at the point of bifurcation, which determines a critical amplitude of the external magnetic field. No equilibrium islands could occur for an external current below this critical value. The point of bifurcation is determined from the condition

$$
\left.\frac{d P(g, \zeta)}{d g}\right|_{g=g_{c r}}=0,
$$

which gives the following equation for the critical $g_{c r}$

$$
4 g^{10}+4 g^{7}-8 g^{4}-6 \zeta^{2}=0 .
$$

The function $P\left(g_{c r}, \zeta\right)$ is shown in Fig. 3 versus the anomalous viscosity parameter $\zeta$. The critical amplitude of an external magnetic field leading to appearance of magnetic island is given by the equation

$$
\begin{aligned}
\left|\frac{\tilde{B}_{r}^{v}}{B_{0}}\right|_{r=a}= & \frac{n S}{2^{8 / 3}} \frac{r_{s}}{a}\left(\frac{r_{s}}{R}\right)\left|\frac{F_{+}(a)\left(-r_{s} \Delta_{-}^{\prime}\right)^{1 / 3}}{r_{s}\left(\Delta_{-}^{\prime}-\Delta_{+}^{\prime}\right)}\right| \\
& \times T_{A}^{4 / 3} \sqrt{P\left(g_{c r}(\zeta), \zeta\right)} .
\end{aligned}
$$

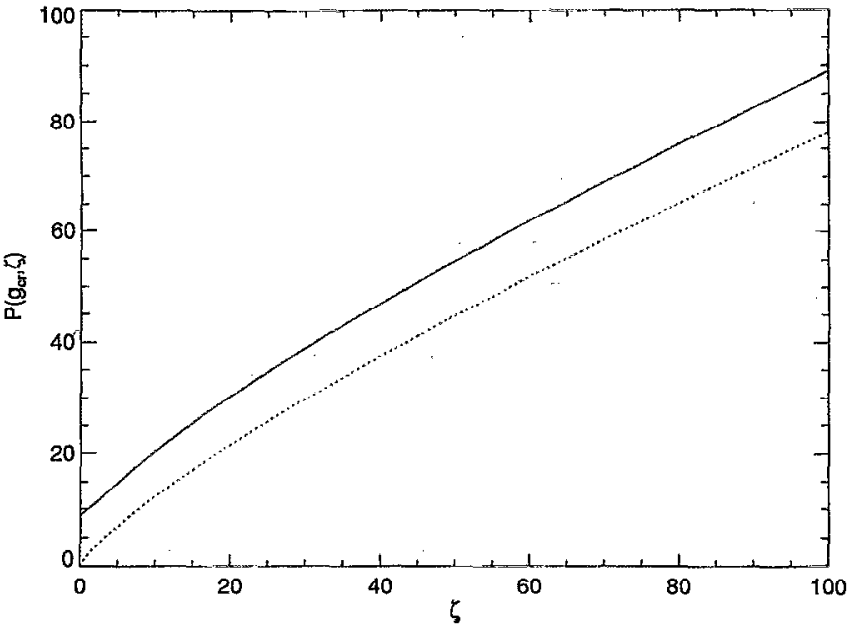

FIG. 3. The function $P\left[g_{c r}(\zeta), \zeta\right]$ versus the $\zeta$ parameter. The critical magnetic field is proportional to $\sqrt{P\left[g_{c r}(\zeta), \zeta\right]}$; solid line-the exact form of $P\left[g_{c r}(\zeta), \zeta\right]$ from Eqs. (103) and (106); dotted line-approximation given by Eq. (109).

In the limit of small anomalous plasma viscosity $[\zeta \ll 1$, $g \rightarrow 1$ and $\left.\sqrt{P\left(g_{c r}, \zeta\right)} \rightarrow 3\right]$, the plasma inertia in the island region is the main stabilizing factor and the expression for the critical magnetic field takes the form

$$
\begin{aligned}
\left|\frac{\tilde{B}_{r}^{v}}{B_{0}}\right|_{r=a}= & \frac{3 G_{3}^{2 / 3}}{2^{4 / 3}} \frac{r_{s}}{a} q^{4 / 3}\left(\frac{r_{s}}{R}\right)^{1 / 3}(n S)^{-1 / 3}\left(-r_{s} \Delta_{-}^{\prime}\right)^{1 / 3} \\
& \times\left|\frac{F_{+}(a)}{r_{s}\left(\Delta_{-}^{\prime}-\Delta_{+}^{\prime}\right)}\right|\left(\tau_{A} \omega_{T}\right)^{4 / 3}
\end{aligned}
$$

In the limit of large viscosity $(\zeta \geqslant 1)$, the solution of Eq. (106) can be approximated by $g_{c r}^{5} \rightarrow \sqrt{3 / 2} \zeta$, and the function $P\left(g_{c r}, \zeta\right)$ in this limit is given by

$$
P\left(g_{c r}, \zeta\right) \rightarrow \frac{5}{3}\left(\frac{3}{2}\right)^{2 / 5} \zeta^{4 / 5}
$$

Then the critical magnetic field is determined by the relation

$$
\begin{aligned}
\left|\frac{\tilde{B}_{r}^{v}}{B_{0}}\right|_{r=a} \simeq & \frac{r_{s}}{a}\left(\frac{r_{s}}{R}\right)^{1 / 5}(n S)^{1 / 5} q^{4 / 5}\left(-r_{s} \Delta_{-}^{\prime}\right)^{3 / 15} \\
& \times\left|\frac{F_{+}(a)}{r_{s}\left(\Delta_{-}^{\prime}-\Delta_{+}^{\prime}\right)}\right|\left(\frac{\tau_{A}^{2} \omega_{T}}{\tau_{M}}\right)^{2 / 5} .
\end{aligned}
$$

The viscous drag always acts so as to increase the critical magnetic field for the nonlinear excitation of a magnetic island. The anomalous plasma viscosity causes also a fite phase shift of the driven magnetic island with respect to the applied resonant magnetic field. It is given by

$$
\tan \Delta \Phi_{0}=\frac{\zeta}{g_{c r}^{2}\left(2+g_{c r}^{3}\right)} .
$$

There is no phase shift if viscosity is ignored, $\Delta \Phi_{0} \rightarrow \pi$ as $\tau_{M} \rightarrow \infty$. For large viscosity $\left(\tau_{M} \rightarrow 0, \zeta \gg 1\right)$, the phase shift increases with $\zeta$ and saturates at the value $\Delta \Phi_{0}=\sqrt{2 / 3}$ for $\zeta>1$. The expressions $(108)$ and $(110)$ are similar in structure to ones obtained previously ${ }^{18}$ except for an additional factor $a^{2} R^{2} / r^{2}$. due to the toroidal enhancement of the ner- 
pendicular inertia (Section III A), which has been phenomenologically introduced in Refs. 18 and 46. Our expressions (108) and (110) also take into account the effects of a finite current density in the region $r_{s}<r<a$.

Note that the criteria (108) and (110) are given in terms of the amplitude of error magnetic field measured $\tilde{B}_{r}^{v}$ in vacuo. The amplitude of the error magnetic field $\tilde{B}_{r}^{\text {cxt }}$ registered at the plasma boundary in the presence of a plasma is different from the $\tilde{B}_{r}^{v}$. It is created by the total magnetic flux $\Psi_{\text {ext }}[$ see Eq. (61)] associated with external currents:

$$
\tilde{B}_{r}^{e x t}=-\left.\frac{1}{a} \frac{\partial}{\partial \theta} \Psi^{e x t}\right|_{r=a},
$$

and related to the vacuum field $\tilde{B}_{r}^{v}$ by the equation

$$
\frac{\left|\tilde{B}_{r}^{e x t}\right|}{\left|\tilde{B}_{r}^{v}\right|}=\left|1-\frac{F_{-}(a)}{F_{+}(a)}\right| \text {. }
$$

Considering the structure of the nonlinear evolution equations (88) and (89), one observes that, in general, there exist two characteristic time scales for the dissipative dynamics of the island width and the rotation frequency. They are given by the resistive diffusion time $\tau_{D}$ and the anomalous viscous damping time $\tau_{M}$. However, the evolution of the island phase may also occur on a much shorter time scale comparable to the ideal Alfvén time, $\tau_{A}$. The fast phase evolution on this time scale could lead to the phase instability, ${ }^{8}$ making the critical equilibrium unstable and thus practically inaccessible. We investigate the stability of the stationary point (102) against small phase oscillations on the ideal MHD time scale. In this regime we obtain from (89) the following equation for the phase evolution:

$$
\begin{aligned}
& 4 \sqrt{2} G_{W}\left(\frac{q}{n S}\right)^{2}\left(\frac{R}{r_{s}}\right)^{2} \tau_{A}^{2} \frac{\partial^{2}}{\partial t^{2}} \Delta \tilde{\Phi} \\
& =-\Gamma^{2} W_{v}^{2} W^{2} r_{s} \Delta_{-}^{\prime} \cos \left(\Delta \Phi_{0}\right) \Delta \tilde{\Phi} .
\end{aligned}
$$

Since $r_{s} \Delta_{\sim}^{\prime} \cos \left(\Delta \Phi_{0}\right)>0$ for the externally driven magnetic island [see Eq. (99)], such an equilibrium is stable with respect to a phase instability; this in contrast to the case of an initially unstable mode suppressed by an external magnetic field. In the latter case, the developing phase instability makes the equilibrium with the suppressed magnetic island unstable, ${ }^{8}$ which, in general, requires a phase tracking for successful realization of feedback stabilization. It is worth noting that the fast evolution of the phase of the magnetic island on the ideal MHD time scale does not mean the magnetic reconnection on the same time scale; the evolution of the magnetic island width occurs on the much slower resistive time scale.

\section{DISCUSSION AND SUMMARY}

We have addressed in this work the problem of the dynamic behaviour of nonlinear tearing modes in a rotating tokamak plasma. Our main goal was to derive fully timedependent nonlinear equations, which can be used for theoretical modeling of the island dynamics both in the feedback stabilization experiments ${ }^{8}$ and in the study of error-fieldinduced instabilities. ${ }^{13}$ We have derived such equations (31) and (25), which govern the evolution of the magnetic island in the presence of an applied resonant magnetic perturbation.

We have taken into our consideration an effect of the ion FLR. As has been shown in Ref. 11, in a slab model ion FLR leads to a modification of the inertia term in the equation (17) for the island width growth, $\left(\omega-\omega_{E}\right)^{2} \rightarrow\left(\omega-\omega_{E}\right)$ $\times\left(\omega-\omega_{E}-\omega_{* i}\right)$. (The effect of the constant radial electric field is easily introduced by transforming to the moving refrence frame, $\omega \rightarrow \omega^{\prime}=\omega-\omega_{E}$.) This modification is exactly the same as for the linear drift-Alfvén waves ${ }^{26}$ in a slab plasma. For the toroidal case [see Eq. (25)], the inertial term is modified as follows: $\left(\omega-\omega_{E}\right)^{2} \rightarrow\left(\omega-\omega_{E}\right)\left(\omega-\omega_{T}\right)$, where $\omega_{T}$ is the frequency of the toroidal plasma rotation determined by the neociassical poloidal flow damping effects. The FLR correction to the inertial term in Eq. (25) could change its sign, so that the effect of inertia becomes destabilizing instead of stabilizing for frequencies in the range $0<\omega^{\prime}<\omega_{* i}+k \omega_{* r}$. Typically, for nonrotating modes induced by static error field perturbations, the effect of plasma inertia is stabilizing. Nonrotating magnetic islands can be destabilized by ion FLR effects by inverting the profile of the radial electric field, so that the diamagnetic flow due to the gradients in the plasma density and temperature is almost compensated by the $\mathbf{E} \times \mathbf{B}$ flow. The corresponding condition for reversal of the sign of the inertial effect from stabilizing to destabilizing is $\Phi_{0}^{\prime}\left(e \Phi_{0}^{\prime} / T_{i}+p_{* i}^{\prime} / p_{0 i}\right.$ $\left.+k T_{0 i}^{\gamma} / T_{0 i}\right)<0$. This situation might be relevant to the $o b-$ served asymmetry in the amplitude of the critical magnetic field in tokamak discharges with "tangential" and/or "perpendicular" neutral beams. ${ }^{14}$

Our nonlinear model takes into account toroidal renormalization of the effective plasma inertia, an effect that has been considered only phenomenologically in previous models. ${ }^{18,46}$ This renormalization comes from the coupling of the longitudinal and perpendicular plasma flows and neoclassical damping of the poloidal plasma velocity in toroidal geometry, and leads to an increase of the inertial term in Eq. (25) by the $q^{2} R^{2} / r^{2}$ factor compared to the slab model (17). Accordingly, the magnitude of the critical error magnetic field leading to a nonlinear excitation of magnetic islands is also increased (see Section V B). Note that this enhancement is partially compensated by the destabilizing effect of perturbed bootstrap current, which may result in the lower amplitude of the critical magnetic field in the regimes with higher values of plasma pressure. ${ }^{14}$

Our toroidal version of the current closure equation (32) is fully consistent with the heuristic momentum balance equation (28). As noted above, the slab equation (6) underestimates the inertia and viscosity terms and is not consistent with the toroidal momentum balance given by Eq. (28). It must be noted that the renormalization of the perpendicular plasma inertia essentially is a result of damping of the poloidal plasma rotation due to neoclassical parallel viscosity, so that expression (22) can be used for a stationary poloidal rotation. which is established on an ion-ion collision time (for the banana collisionality regime). In the regimes where the characteristic time scale for an island evolution is shorter or comparable to the ion-ion collision time, the timedependent equation for the poloidal rotation has to be solved 
and effects of the toroidal coupling of poloidal and toroidal flows and damping of polodal rotation are expected to be modified significantly. An external electromagnetic torque also could modify the poloidal momentum balance condition. Consideration of all these effects requires new approaches for calculation of the time-dependent unaveraged neoclassical viscosity, ${ }^{56}$ and is out of the scope of the present work.

There are several mechanisms of slowing down for the rotation of magnetic islands in a tokamak. In addition to effects of mode interaction with eddy currents in a resistive wall, ${ }^{10,46,51,57}$ we have identified several other mechanisms of reduction in the mode rotation. Our equation (89) for the evolution of the rotation frequency shows that in the absence of an interaction with the wall the mode rotation slows down to the $\omega_{T}$ frequency due to viscous transfer of the toroidal momentum from the island region to the bulk plasma. Thus, for the magnetic islands that are in equilibrium with the ion component of a tokamak plasma (through the anomalous ion viscosity), $\omega_{T}$ is the "natural" frequency of mode rotation. The effects of plasma inertia, viscosity and bootstrap current drive become less important for magnetic islands as they grow in size. Such a situation is expected for naturally occurring magnetic islands such as those observed in the TEXT tokamak. ${ }^{20}$ In this case the inertial and viscous effects can be neglected, and the momentum of the rotating plasma is constant. Then, the island rotation slows down as the island grows and the rotation frequency decreases inversely with the island width [see Eq. (85)]. Such an effect is probably most important for the magnetic islands in the Rutherford regime. There is also additional damping of the toroidal rotation of a magnetic island due to a neoclassical toroidal viscous force associated with destroyed axial symmetry of the magnetic configuration with the imposed helical magnetic field (Section III B). This effect is probably negligible in present experiments, but could be important in larger tokamaks.

We have analyzed the structure of the magnetic flux perturbation in the "linear" (or ideal MHD response) region, assuming an arbitrary time dependence for both the external field and the reconnected flux, bearing in mind a possible application to the problem of feedback stabilization of tearing modes. The effect of finite current density between the rational surface and the plasma boundary has been included in this analysis. It has been shown that the magnetic perturbation in the outer region (and, in particular, that registered outside the plasma) consists of several different components [see Eq. (55)]. The main part of the magnetic flux in this region is directly related to the magnetic island and created by the finite value of the reconnected flux at the rational surface [the first term in Eq. (55)]. Another part, which exactly vanishes at the rational surface, is induced by the external current [the second term in Eq. (55)]. Within the framework of linear theory these two components are independent and have arbitrary rotation frequencies. The third part of the magnetic perturbation in the linear region corresponds to the part of the magnetic flux associated with the first two components reflected from the resistive wall [the last term in Eq. (55)]. The corresponding components of the magnetic flux have a finite phase shift, which is determined by the wall resistivity, with respect to their counterparts [see Eqs. (68), (69), (75) and (76)].

In the absence of the stabilizing effects of plasma inertia and quasilinear saturation, ${ }^{58}$ any external resonant magnetic perturbation would lead to excitation of magnetic islands with a finite amplitude in a stable plasma. The effect of plasma inertia in the island region stabilizes magnetic islands at small amplitude, thus establishing a critical value for the external magnetic field required to produce a nonlinear magnetic island. In general, this critical magnetic field is a function of plasma inertia (which can be parametrized by the characteristic poloidal Alfvén time), and the plasma rotation damping rate due to anomalous plasma viscosity. In the limit of small viscosity, the critical amplitude of an external field is determined mainly by the inertial effect, while in the opposite limit, both effects become comparable. The basic structure of our nonlinear evolution equations is similar to that obtained in Ref. 12 apart from the above mentioned toroidal (neoclassical) and ion FLR effects. The anomalous plasma viscosity enhances a critical magnetic field required to induce a magnetic island. It is worth noting that the increase of the critical magnetic field due to anomalous perpendicular plasma viscosity is directly related to a finite phase shift (111) between the applied magnetic perturbation and the induced magnetic island. This phase shift is similar to that created by the resistive wall [see Eqs. (68) and (69)]. Note that a similar effect would be created by finite plasma resistivity in the outer region $r_{s}<r<a, e . g$., by the cold plasma at the periphery of a plasma column. Comparison of the experimental data on the phase location of the locked mode with respect to the phase of an external current with predictions of the present model, as given by Eq. (111), would help to determine whether viscosity effects are important in the dynamics of locked modes. The relative importance of the anomalous viscosity effect is difficult to predict with high reliability now, since only indirect measurements of the anomalous viscosity are available. The uncertainties are too large in the existing experimental data on the nonlinear excitation of a magnetic island by an externally applied resonant magnetic perturbation to conclude whether the present tokamaks are in the inertial or viscosity dominated regime. It has been suggested in Ref. 14 that the critical magnetic ficld is determined by the viscous drag force, while in Refs. 6 and 7 it was assumed to be determined mainly by inertial effects, with a weak dependence on the anomalous plasma viscosity. Note that the scalings for the inertia and viscosity dominated regimes are quite different from each other ${ }^{6,7,14}$ [see also Eqs. (108) and (110)]. If we assume that the anomalous plasma viscosity coefficient is of the order of the gyro-Bohm diffusion coefficient, then the parameter $\zeta$ characterizing viscosity effect can be approximated as $\zeta \approx\left(a / \rho_{i}\right)^{1 / 3}\left(\epsilon^{4} / \beta\right)^{2 / 3}$. It is worth noting that the expression (110) for the critical magnetic field gives good approximation to the exact form (107) only for significantly large values of the anomalous plasma viscosity, as is illustrated in Fig. 3, where both the exact function $P\left(g_{c r}, \zeta\right)$ and approximation (109) are shown.

Although in the present paper we concentrated on the 
mechanism of a nonlinear bifurcation into a state with a finite island width from the initial equilibrium without magnetic islands, our nonlinear equations (88) and (89) can be used as well for nonlinear modeling of mode locking for initially rotating magnetic islands with a finite island width. We presume here that magnetic islands, which are usually observed in tokamak experiments (in particular, those associated with locked modes), have a size larger than the linear inertial tearing layer width, so that they are in the nonlinear regime, and the Rutherford-type theory can be used to describe of their dynamics. ${ }^{9}$ The interaction of an external field with magnetic perturbations in a suppressed state (before entering the nonlinear Rutherford regime) was discussed in Ref. 59.

In the present paper we have not discussed the further evolution after the appearance of locked modes. So far it is unclear what is the cause of the sudden growth of the magnetic island that is usually observed after mode locking. ${ }^{17}$ It has been suggested that it is related to the loss of the wall stabilization for nonrotating modes ${ }^{50}$ which is associated with the change in the effective $\Delta^{\prime}$ parameter. Locking of the mode rotation into the reference frame of a static error field, and subsequent destabilization of the mode growth can be avoided by applying an external helical magnetic field rotating with a constant frequency and an amplitude slightly larger than the level of error fields. ${ }^{34}$ Then, the induced magnetic islands would have to lock into the rotating external field. Such locked rotating islands should have smaller amplitude, because the resistive wall stabilization is still in effect for them. Experiments with a rotating external helical field and comparison to similar ones with a nonrotating field would help to identify whether an additional destabilization mechanism such as the neoclassical bootstrap current ${ }^{30}$ is required to explain the size of the magnetic islands observed in experiments.

\section{ACKNOWLEDGMENTS}

Helpful discussions with Dr. L. E. Zakharov and Dr. C. C. Hegna are acknowledged with gratitude.

This work supported by the Natural Sciences and Engineering Research Council and National Fusion Program of Canada, Consiglio Nazionale delle Ricerche of Italy and United States Department of Energy Grant No. DE-FG02-86 ER53218.

\section{APPENDIX: THE PERTURBED PLASMA DENSITY AND ELECTROSTATIC POTENTIAL NEAR MAGNETIC ISLAND}

The perturbation in the plasma density and electrostatic potential associated with the moving magnetic perturbation are determined from the equations of two-fluid magnetohydrodynamics. The electron density is found from the parallel component of the electron momentum balance equation, and the ion density is determined from the continuity equation. The quasineutrality condition is used to find the electrostatic potential. In the steady-state approximation, the electron momentum balance equation has the form

$$
-\nabla_{\mid} \Phi+\frac{\omega}{c} \tilde{\psi} \sin \xi+\frac{T_{e}}{e n_{0}} \nabla_{\mid} n=0 .
$$

Inverting operator (11) with identity (12), we obtain

$$
n_{e}=\frac{e n_{0}}{T_{0 e}}\left(\Phi-\frac{\omega B_{0}}{k_{\theta} c} x\right)+F(\psi),
$$

where the function $F(\psi)$ is the "constant" of integration. Separating the total electrostatic potential $\Phi$ into the equilibrium and perturbed part,

$$
\Phi=\Phi_{0}^{\prime} x+\tilde{\Phi},
$$

one can rewrite Eq. (A2) in the form

$n_{e}=\frac{e n_{0}}{T_{0 e}}\left(\tilde{\Phi}-\frac{\left(\omega-\omega_{E}\right) B_{0}}{k_{\theta} c} x+\frac{B_{0}}{k_{\theta} c}\left(\omega-\omega_{E}-\omega_{* e}\right) \lambda(\psi)\right)$.

Here, the $\lambda(\psi)$ function is introduced by the relation

$$
F(\psi)=\frac{e n_{0}}{T_{0 e}} \frac{B_{0}}{k_{\theta} c}\left(\omega-\omega_{E}-\omega_{* e}\right) \lambda(\psi),
$$

where $\omega_{* e}=-k_{\theta} c T_{0 e} n_{0}^{\prime} / e B_{0} n_{0}$ is the electron diamagnetic frequency.

The ion continuity equation gives, after integration, ${ }^{19}$

$$
n_{i}=n_{0}^{\prime}\left(x-\frac{k_{\theta} c}{\left(\omega-\omega_{E}\right) B_{0}} \tilde{\Phi}\right)+\frac{c^{2}}{4 \pi e v_{A}^{2}}\left(1-\frac{\omega_{* i}}{\omega-\omega_{E}}\right) \nabla_{\perp}^{2} \tilde{\Phi}
$$

Using the quasineutrality condition $n_{i}=n_{e}$, we obtain the equation for the perturbed electrostatic potential $\tilde{\Phi}$,

$$
\begin{aligned}
\tilde{\Phi}\left(1-\frac{\omega_{* e}}{\omega-\omega_{E}}\right)= & \frac{B_{0}}{k \theta c}\left(\omega-\omega_{E}-\omega_{* e}\right)[x-\lambda(\psi)] \\
& \times \frac{c^{2}}{4 \pi e v_{A}^{2}} \frac{T_{0 e}}{e n_{0}}\left(1-\frac{\omega_{* i}}{\omega-\omega_{E}}\right) \nabla_{\perp}^{2} \tilde{\Phi} .
\end{aligned}
$$

In the approximation of large magnetic island width, $W \gg \rho_{i}$, the last term on the right of this equation can be neglected, and we obtain in the leading order Eq. (14) for the perturbed electrostatic potential. We have assumed equilibrium boundary conditions in Eqss. (A2), (A6), and (A7), so that $n_{i} \rightarrow n_{0}^{\prime} x$. The boundary conditions for the density and electrostatic potential mean that for large $x(\gg W)$ the perturbation caused by the magnetic island decays, so that $\tilde{\Phi} \rightarrow 0$, and the equilibrium value of the plasma density is recovered, $n \rightarrow n_{0}^{\prime} x$. Then, the function $\lambda(\psi)$ in (A5) asymptotically be given by

$$
\lambda(\psi) \rightarrow x
$$

for large $x \gg W$. The proper choice of the function $\lambda(\psi)$ assumes also that in the limit $x \geqslant W$, the usual linear relationships between $n, J_{\|}, \Phi$ and $\tilde{\psi}$ can be recovered from the nonlinear expressions (A4), (A6), and (16). The simplest choice of the function $\lambda(\psi)$ that satisfies these conditions is

$$
\lambda(\psi)=\frac{W}{\sqrt{2}}\left[\left(-\frac{\psi}{\tilde{\psi}}\right)^{1 / 2}-1\right] .
$$


As a matter of fact, the profile of the plasma density, temperature and electrostatic potential should be determined from the solution of the corresponding transport equations in the magnetic island geometry. For instance, in the limit of large heat flux across the magnetic surfaces, the plasma temperature can be found from the heat diffusivity equation. ${ }^{9}$ As was noted in Ref. 30, the different profiles of the plasma pressure in Eqs. (A4) and (A9) give quantitatively the same results with slightly different numerical coefficients. It is worth noting that the profiles of plasma parameters such as density, temperature, electrostatic potential and electric current are specified by the $\lambda(\psi)$ function (A9), which is determined only by linear boundary conditions at $x \gg w$. Such an $\lambda(\psi)$ function is ill defined at the island separatrix, which causes some singularities in the longitudinal current density at the separatrix, although these singularities do not lead to singularites in the total current. The authors of Ref. 12 paid special attention to this problem and have removed the singularities by means of a rather complicated procedure of asymptotic matching across the magnetic separatrix. Though this procedure is much more complicated than ours used in Refs. 11 and 19, it turns out that for the inertial term the only difference is in the numerical coefficient $G_{3}$, which is 0.96 in Ref. 12 and 1.06 in our work ${ }^{11}$ (in the same notation). Thus, we assume that the simple form of the $\lambda(\psi)$ function given by Eq. (A9) provides reasonable estimates for the effects considered in this work. In general, the profile functions for the density, temperature, etc. could be different, because of different physical phenomena involved in the transport processes. Since the physics of the transport in a tokamak is not very well determined at present, we will use the same function (A9) for the density, temperature and potential profiles.

IA.W. Morris, Plasma Phys. Controlled Fusion 34, 1871 (1992).

${ }^{2}$ J.T. Scoville R.J. La Haye, A.G. Kellman, T.H. Osborne, R.D. Stambaugh, E.J. Strait, and T.S. Taylor, Nucl. Fusion 31, 875 (1991).

${ }^{3}$ V.V. Volkov, A.A. Gurov, N.V. Ivanov, A.M. Kakurin, and D.A. Martynov, Sov. J. Plasma Phys. 17, 7 (1991).

${ }^{4}$ A.W. Morris, Phys. Rev. Lett. 64, 1254 (1990).

${ }^{5}$ Yu.V. Gvozdkov, N.V. Ivanov, and A.M. Kakurin, Sov. J. Plasma Phys. 6, $130(1980)$

${ }^{6}$ R.J. La Haye, R. Fitzpatrick, T.C. Hender, A.W. Morris, J.T. Scoville, and T.N. Todd, Phys. Fluids B 4, 2098 (1992).

${ }^{7}$ See National Technical Information Service Document No. DE 93014408/LL ( R.J. La Haye, Limits on $m=2, n=1$ error field induced locked mode instability in TPX with typical sources of poloidal field coil error field and a prototype correction coil, "C-coil", General Atomics Report No. GA-A21167, UC-420, December 1992). Copies may be ordered from National Technical Information Service, Springfield, Virginia 22161.

${ }^{s}$ E. Lazzaro and M.F.F. Nave, Phys. Fluids 31, 1623 (1988).

${ }^{9}$ P.H. Rutherford, Phys. Fluids 16, 1903 (1973).

${ }^{10}$ P.H. Rutherford, Proceedings of the Course and Workshop on Basic Physical Processes of Toroidal Fusion Plasmas (Commission of the European Communities, Brussels, 1985), Vol. 2, p. 531.

"A.I. Smolyakov, Sov. J. Plasma Phys. 15, 667 (1989).

${ }^{12}$ R. Fitzpatrick and T.C. Hender, Phys. Fluids B 3, 644 (1991).

${ }^{13}$ G.M. Fishpool and P.S. Haynes, Nucl. Fusion 34, 109 (1994).

${ }^{14}$ R.J. La Haye, A.W. Hyatt, and J.T. Scoville, Nucl. Fusion 32, 2119 (1992).

${ }^{15}$ A.W. Morris, P.G. Carolan, R. Fitzpatrick, T.C. Hender, and T.D. Todd, Phys. Fluids B 4, 413 (1992).

${ }^{16}$ T.N. Todd, P.G. Carolan, J.W. Connor, R. Fitzpatrick, R.J. Hastie, I.C. Hender, A.W. Morris, the COMPASS Team, T. Tuda, G. Kurita, M. Azumi, T. Takeda, R.J. La Haye, A.W. Hyatt, and J.T. Scoville, in Plasma Physics and Controlled Nuclear Fusion Research, Würzburg, 1992 (International Atomic Energy Agency, Vienna, 1993), Vol. 2, p. 3.

${ }^{17}$ J.A. Snipes, D.J. Campbell, P.S. Haynes, T.C. Hender, M. Hugon, P.J. Lomas, N.J. Lopez Cardozo, M.F.F. Nave, and F.C. Schüller, Nucl. Fusion 28, 1085 (1988).

${ }^{18}$ T.C. Hender, R. Fitzpatrick, A.W. Morris, P.G. Carolan, R.D. Durst, T. Edlington, J. Ferreira, S.J. Fielding, P.S. Haynes, J. Hugill, I.J. Jenkins, R.J. La Haye, B.J. Parham, D.C. Robinson, T.N. Todd, M. Valovic, and G. Vayakis, Nucl. Fusion 32, 2091 (1992).

${ }^{19}$ A.I. Smolyakov, Plasma Phys. Controlled Fusion 35, 657 (1993).

${ }^{20}$ D.L. Brower, S.C. McCool, J.Y. Chen, A.J. Wootton, C.X. Yu, R.V. Bravenec, G.G. Castle, K.W. Gentle, R.L. Hickok, Y. Jiang, G.X. Li, H. Lin, N.C. Luhmann, Jr., J.B. Tuylor, B.Z. Zhang, and S.J. Zhao, in Ref. 16, p. 177.

${ }^{21}$ J.D. Callen, W.X. Qu, K.D. Siebert, B.A. Carreras, K.C. Shaing, and D.A. Spong, in Plasma Physics and Controlled Nuclear Fusion Research 1986, Kyoto (International Atomic Energy Agency, Vienna, 1987), Vol. 2, p. 157.

${ }^{22}$ T.H. Jensen, A.W. Leonardo, R.J. La Haye, and M.S. Chu, Phys. Fluids B 3, 1650 (1991).

${ }^{23}$ T.H. Jensen, A.W. Leonardo, and A.W. Hyatt, Phys. Fluids B 5, 1239 (1993).

${ }^{24}$ D. Edery, M. Frey, J.D. Somon, M. Tagger, J.L. Soule, R. Pellat, and M.N. Bussac, Phys. Fluids 26, 1165 (1983).

${ }^{25}$ B. Scott and A.B. Hassam, Phys. Fluids B 3, 1650 (1991).

${ }^{26}$ A.B. Mikhailovskii,, Theory of Plasma Instabilities (Consultants Bureau, New York, 1974), Vol. 2.

${ }^{27}$ D. Biskamp, Nucl. Fusion 18, 1059 (1978).

${ }^{28}$ S.I. Braginskii, in Reviews of Plasma Physics, edited by M. Leontovich (Consultants Bureau, New York, 1965), Vol. 1.

${ }^{29}$ S. P. Hirshman and D. J. Sigmar, Nucl. Fusion 21, 1079 (1981).

${ }^{30}$ R. Carrera, R.D. Hazeltine, and M. Kotschenreuther, Phys. Fluids 29, 899 (1986); C.C. Hegna and J.D. Callen, Phys. Fluids B 4, 1855 (1992); also see National Technical Information Service Document No. DE 86008946/LL (W.X. Qu and J.D. Callen, Nonlinear Growth of a Single Neoclassical MHD Tearing Mode in a Tokamak, University of Wisconsin Plasma Report, No. UWPR 85-5). Copies may be ordered from National Technical Information Service, Springfield, Virginia 22161.

${ }^{31}$ T.H. Jensen and M.S. Chu, J. Plasma Phys. 30, 57 (1983).

${ }^{32}$ A.H. Nayfeh, Introduction to Perturbation Techniques (Wiley, New York, 1981) p. 388.

${ }^{33}$ D. Edery and A. Samain, Plasma Phys. Controlled Fusion 32, 93 (1990).

${ }^{34} \mathrm{G}$. Kurita, T. Tuda, M. Azumi, and T. Takeda, Nucl. Fusion 32, 1899 (1992).

${ }^{35}$ W. Mao and M. Wang, Phys. Plasmas 1, 946 (1994)

${ }^{36} \mathrm{H}$. Zohm, A. Kallenbach, H. Bruhns, G. Fussmann, and O. Klüber, Europhys. Lett. 11, 745 (1990).

${ }^{37}$ R.J. La Haye, R.J. Groebner, A.W. Hyatt, and J.T. Scoville, Nucl. Fusion 33, 349 (1993).

${ }^{38}$ P.V. Savrukhin, E.S. Lyadina, D.A. Martynov, D.A. Kislov, and V.I. Poznyak, Nucl. Fusion 34, 317 (1994).

${ }^{39}$ J.W. Connor, S.C. Cowley, R.J. Hastie, T.C. Hender, A. Hood, and T.J. Martin, Phys. Fluids B 3, 70 (1988).

${ }^{40}$ M. Persson, R.L. Dewar, and E.K. Maschke, Phys. Fluids B 5, 3844 (1993).

${ }^{41}$ R. Fitzpatrick, Phys. Plasmas 1, 3308 (1994).

${ }^{42}$ Z. Chang and J.D. Callen, Nucl. Fusion 30, 219 (1990).

${ }^{43}$ K.C. Shaing, S.P. Hirshman, and J.D. Callen, Phys. Fluids 29, 521 (1986).

${ }^{44}$ J.A. Wesson, R.D. Hill, M. Hugon, F.C. Schüller, J.A. Snipes, D.J. Ward, D.V. Bartlett, D.J. Campbell, P.A. Duperrex, A.W. Edwards, R.S. Granetz, N.A.O. Cottardi, T.C. Hender, E. Lazzaro, P.J. Lomas, N. Lopez Cardozo, K.F. Mast, M.F.F. Nave, N.A. Salman, P. Smeulders, P.R. Thomas, B.J.D. Tubbing, M.F. Turner, and A.Weller, Nucl. Fusion 29, 641 (1989).

${ }^{45}$ J.A. Wesson, Nucl. Fusion 18, 87 (1978).

${ }^{46}$ M.F.F. Nave and J.A. Wesson, Nucl. Fusion 30, 2575 (1990).

${ }^{47}$ C.G. Gimblett, Nucl. Fusion 26, 617 (1986).

${ }^{48}$ B.N. Kuvshinov, Sov. J. Plasma Phys. 16, 642 (1990); L.E. Zakharov (private communication, 1982).

${ }^{49}$ T.S. Hahm and R.M. Kulsrud, Phys. Fluids 28, 2412 (1985).

${ }^{50} \mathrm{~A}$. Bondeson and M. Persson, Nucl. Fusion 28, 1887 (1988).

${ }^{51}$ T.C. Hender, C.G. Gimblett, and D.C. Robinson, Nucl. Fusion 29, 1279 (1989). 
${ }^{52}$ G.A. Hallock, J. Mathew, W.C. Jennings, R.L. Hickok, A.J. Wootton, and R.C. Isler, Phys. Rev, Lett. 56, 1248 (1986).

${ }^{53}$ K.W. Gentle, Nucl. Technol,/Fusion 1, 479 (1981).

${ }^{54}$ A. Reiman, Phys. Fluids B 3, 2617 (1991): A. Reiman and D. Monticello. ibid. 3, 2230 (1991).

${ }^{55} \mathrm{~J} . \mathrm{K}$. Lee, H. Ikezi, F.W. McClain, and N. Ohyabu, Nucl. Fusion 23, 63 (1983).
${ }^{56}$ J.P. Wang and J.D. Callen, Phys. Fluids B 5, 3207 (1993); C.T. Hsu, K.C. Shaing, and R. Gormley, Phys. Plasmas 1, 132 (1994).

${ }^{57} \mathrm{M}$. Persson and A. Bondeson, Nucl. Fusion 29, 989 (1989).

${ }^{58}$ R.B. White, D.A. Monticello, M.N. Rosenbluth, and B.V. Waddell, Phys. Fluids 20. 800 (1977).

${ }^{59}$ R. Fitzpatrick. Nucl. Fusion 33, 1049 (1993). 